\title{
The Nicotiana benthamiana Mitogen-Activated Protein Kinase Cascade and WRKY Transcription Factor Participate in Nep1 ${ }_{\mathrm{Mo}_{\mathrm{o}}}$-Triggered Plant Responses
}

\author{
Huajian Zhang, Deqing Li, Meifang Wang, Jiewen Liu, Wenjun Teng, Baoping Cheng, Qian Huang, \\ Min Wang, Wenwen Song, Suomeng Dong, Xiaobo Zheng, and Zhengguang Zhang \\ Department of Plant Pathology, College of Plant Protection, Nanjing Agricultural University, Key Laboratory of Monitoring \\ and Management of Crop Diseases and Pest Insects, Ministry of Agriculture, Nanjing, 210095, China
}

Submitted 15 November 2011. Accepted 18 July 2012.

\begin{abstract}
Many bacterial, fungal, and oomycete species secrete necrosis and ethylene-inducing peptide 1 (Nep1)-like proteins (NLP) that trigger programmed cell death (PCD) and innate immune responses in dicotyledonous plants. However, how NLP induce such immune responses is not understood. Here, we show that silencing of the MAPKKK $\alpha-$ MEK2-WIPK mitogen-activated protein kinase (MAPK) cascade through virus-induced gene silencing compromises hydrogen peroxide accumulation and PCD induced by Nep1 $_{\mathrm{Mo}}$ from Magnaporthe oryzae. WIPK interacts with NbWRKY2, a transcription factor in Nicotiana benthamiana, in vitro and in vivo, suggesting an effector pathway that mediates Nep1 $_{M_{0}}$-induced cell death. Unexpectedly, salicylic acid-induced protein kinase (SIPK)- and NbWRKY2silenced plants showed impaired $\mathrm{Nep}_{\mathrm{Mo}}$-induced stomatal closure, decreased Nep1 $1_{M o}$-promoted nitric oxide (NO) production in guard cells, and a reduction in $\mathrm{Nep} 1_{\mathrm{Mo}_{0} \text {-induced }}$ resistance against Phytophthora nicotianae. Expression studies by real-time polymerase chain reaction suggested that the MEK2-WIPK-NbWRKY2 pathway regulated Nep1 $\mathrm{Mo}^{-}$ triggered NO accumulation could be partly dependent on nitrate reductase, which was implicated in NO synthesis. Taken together, these studies demonstrate that the MAPK cascade is involved in $\mathrm{Nep}_{\mathrm{Mo}_{0}}$-triggered plant responses and MAPK signaling associated with PCD exhibits shared and distinct components with that for stomatal closure.
\end{abstract}

Plants have evolved multiple defense mechanisms against phytopathogens, including the hypersensitive response (HR) and stomatal closure (Dangl et al. 1996; Lam et al. 2001; Melotto et al. 2006; Mur et al. 2008). HR is considered a form of localized programmed cell death (PCD) that results in the formation of necrotic lesions around infection sites (Greenberg and Yao 2004; Hammond-Kosack and Jones 1996). The PCD program is initiated upon plant-pathogen recognition. In addition to classic gene-for-gene interactions, bindings of certain selected pathogen-associated molecular patterns (PAMPs) (also called microbe- or danger-associated molecular patterns) and nonhost-

Current address for Huajian Zhang: Department of Plant Pathology, Anhui Agricultural University, Hefei, Anhui, 230026, China.

Corresponding author: Z. Zhang; Telephone: +86-25-84396972; Fax: +8625-84395325; E-mail: zhgzhang@njau.edu.cn

* The $e$-Xtra logo stands for "electronic extra" and indicates that four supplementary figures and one supplementary table are published online. specific elicitors to their receptors can trigger HR (Nürnberger et al. 2004; Schwessinger and Zipfel 2008; Zhang et al. 1998). Plants recognize invading microbes by sensing PAMPs and activate downstream signaling cascades, leading to PAMP-triggered immunity (PTI) (Schwessinger and Zipfel 2008).

Necrosis and ethylene-inducing peptide 1 (Nep1) is secreted by taxonomically diverse organisms, including bacteria, fungi, and oomycetes (Cechin et al. 2008). NLP (Nep1-like protein) from different pathogens play distinct roles in pathogen virulence. In some cases, microbial NLP are considered as positive virulence factors accelerating disease and pathogen growth in host plants through disintegration. The virulence could be increased by transformation of Colletotrichum coccodes with Fusarium oxysporum and the spectrum of hosts could be infected (Amsellem et al. 2002). Also, NLP-deficient mutants of Erwinia carotovora were reduced in virulence on both rubbers and stems of potato (Pemberton et al. 2005). Transformation of the NLP-deficient Pectobacterium carotovoum subsp. carotovorum strain with $\mathrm{NLP}_{\mathrm{Pya}^{-}}$or $\mathrm{NLP}_{\mathrm{Pp}}$-encoding sequences showed partial restoration (30 to $40 \%$ ) in bacterial virulence and potato tuber maceration (Ottman et al. 2009). However, the deletion of NLP in fungal pathogens Botrytis elliptica, Mycosphaerella graminicola, and Verticillium dahliae did not attenuate virulence, suggesting that NLP are not virulence determinants in these plant pathogen interactions (Motteram et al. 2009; Staats et al. 2007; Zhou et al. 2012).

In many different dicotyledonous plants, the recognition of Nep1 results in cell death (Gijzen and Nürnberger 2006; Pemberton and Salmond 2004; Schwessinger and Zipfel 2008). It is suggested that NLP may associate with the outer surface of the plasma membrane to trigger HR (Qutob et al. 2006; Schouten et al. 2008), and global gene expression analyses also revealed an overlap between the responses to NLP and other elicitors leading to ethylene and active oxygen production (Bae et al. 2006; Garcia-Brugger et al. 2006). The mitogenactivated protein kinase (MAPK) cascade is known to play important roles in mediating cellular membrane stress and cell wall-integrity pathways and, therefore, could be important in studying NLP-induced plant immune responses.

The MAPK cascades are major pathways that transduce and amplify extracellular and intracellular stimuli to a wide range of overlapping or specific intracellular responses in eukaryotic cells (Chen and Thorner 2007). The MAPK pathway contains a functionally three-tiered kinase cascade comprising a MAPK kinase kinase (MAPKKK, also called MAP3K, MEKK, or MKKK), a MAPK kinase (MAPKK, also called MAP2K, MEK, or MKK), and a MAPK. Plant MAPK cascades have 
been implicated in diverse biological processes, including cell division, hormone signaling, cell-wall biosynthesis, cell growth and differentiation, abiotic stress, and biotic stress (Andreasson et al. 2005; Ludwig et al. 2005; Takahashi et al. 2004; Teige et al. 2004). Recent studies demonstrated that MAPK are rapidly activated in different eukaryotic pathogens and pathogenderived elicitors or PAMP-triggered plant defense (Asai et al. 2008; del Pozo et al. 2004; Liu et al. 2008; Nakagami et al. 2005; Oh et al. 2009; Pedley and Martin 2005; Ren et al. 2006; Tena et al. 2001; Zhang and Klessig 2001).

Increasing evidence has also revealed that MAPK play key roles in various inducer-triggered hypersensitive cell deaths. Two key MAPK isolated from tobacco (i.e., wound-induced protein kinase [WIPK] and salicylic acid-induced protein kinase [SIPK] $)$ are associated with elicitins and $N$ - and $C f 9$-mediated defense (Jin et al. 2003; Romeis et al. 1999; Zhang and Klessig 1998; Zhang et al. 2000). In the absence of pathogens, expression of $\mathrm{NtMEK} 2^{\mathrm{DD}}$, a constitutively active mutant of MEK2, triggers HR-like cell death, defense gene expression, and active oxygen species (AOS) generation, all of which are preceded by activation of endogenous WIPK and SIPK (Jin et al. 2003). Similarly, heterologous expression of potato (Solanum tuberosum) StMEK2 ${ }^{\mathrm{DD}}$ in Nicotiana benthamiana leads to the activation of WIPK and SIPK (Katou et al. 2003) and generation of AOS (Yoshioka et al. 2003). Potential MAPKKK upstream of NtMEK2-SIPK/Ntf4/WIPK include orthologs of Arabidopsis MEKK1 and tobacco MAPKKK $\alpha$ (Asai et al. 2002; del Pozo et al. 2004). Silencing of components in the MAPKKK $\alpha-$ MEK2-SIPK cascade prevents AvrPto and Pto-mediated hypersensitive cell death (HCD), and MAPKKK $\alpha$ overexpressiontriggered HR-like cell death is attenuated not only by silencing $M E K 2$ or SIPK, but also by silencing MEK1 or NTF6, providing loss-of-function evidence that this MAPK pathway regulates the HR cell-death process (del Pozo et al. 2004; Oh et al. 2009). Consistent with WIPK and SIPK, silencing of NPK1, $M E K 1$, or NTF6 attenuates $N$ - and Pto-mediated resistance to Tobacco mosaic virus (Jin et al. 2002, 2003; Liu et al. 2003; del Pozo et al. 2004) and Pseudomonas syringae, respectively (Ekengren et al. 2003). Treatment of $N$. benthamiana with elicitin INF1 (derived from Phytophthora infestans) activates the MEK2-SIPK/NTF4 cascade to control the NOA1-mediated nitric oxide (NO) burst, and MAPK cascades (MEK2-SIPK/ NTF4 and MEK1-NTF6) regulate the NADPH oxidase-dependent oxidative burst by gain-of-function and loss-of-function analyses, respectively (Asai et al. 2008). Additionally, several WRKY transcription factors have been shown to be phosphorylated by MAPK and to be involved in plant immunity (Eulgem and Somssich 2007; Kim and Zhang 2004; Menke et al. 2005; Popescu et al. 2009).

We previously demonstrated the involvement of respiratory burst oxidase homolog $(\mathrm{RBOH})$, vacuolar processing enzyme (VPE), and G proteins in elicitor-triggered plant immunity by Potato virus $X$ (PVX)-based virus-induced gene silencing (VIGS) (Zhang et al. 2009, 2010, 2012). In the present study, we characterized the roles of the MAPK cascade and WRKY transcription factor in the regulation of $\mathrm{Nep} 1_{\mathrm{Mo}_{\mathrm{o}}}$-induced responses using VIGS. This study will expand our understanding of the signaling networks involved in plant responses to Nep $1_{\mathrm{Mo}}$ and other elicitors.

\section{RESULTS}

\section{Silencing of MAPKKK $\alpha, M E K 2$, and WIPK}

compromises the Nep1 $1_{M o}$-triggered $H R$.

The use of PVX for VIGS in $N$. benthamiana has been demonstrated previously in studies of $\mathrm{RBOH}, \mathrm{VPE}$, and G proteins (Zhang et al. 2009, 2010, 2012). Previous studies sug-

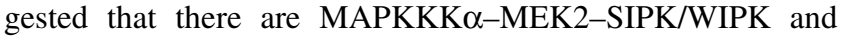
MAPKKK $\alpha-M E K 1-N T F 6$ pathways in $N$. benthamiana (Ekengren et al. 2003; del Pozo et al. 2004; Pedley and Martin 2004). Complementary DNA fragments corresponding to $M A P K K K \alpha, M E K 1, M E K 2, W I P K$, SIPK, and NTF6 were isolated from $N$. benthamiana and were cloned into PVX binary vector pGR107 (PVX-MAPKKK $\alpha$, PVX-MEK1, PVX-MEK2, PVX-WIPK, PVX-SIPK, or PVX-NTF6) and were then introduced into $N$. benthamiana by Agrobacterium infiltration (Ekendren et al. 2003; del Pozo et al. 2004; Liu et al. 2004). Three to four weeks after PVX infection, RNA was isolated from leaf tissue collected from the upper part of each of the silenced plants. For each gene-silenced and PVX-only infected plant, the relative abundance of transcripts for the targeted gene was determined by semiquantitative reverse transcription-polymerase chain reaction (RT-PCR) (Fig. 1A). As expected, the transcript of each gene tested decreased markedly in the silenced plants compared with the control plants infected with the PVX vector alone. Moreover, both real-time PCR and Western immunoblot analysis confirmed that silencing of $M E K 1, M E K 2$, WIPK, SIPK, and NTF6 was specific with no co-silencing occurring (Supplementary Fig. S1).

We next investigated the possible involvement of each of the signaling genes in $\mathrm{Nep} 1_{\mathrm{Mo}}$-mediated cell death. Three to four weeks after viral infection, the silenced leaves verified by RTPCR were inoculated with Nep $1_{\text {Mo }}$ solution or phosphate-buffered saline (PBS) buffer as a control, and the reactions of different plants to Nep1 $1_{\text {Mo }}$ were compared (Supplementary Fig. $\mathrm{S} 2$ ). Infiltration of the Nep1 $1_{\mathrm{Mo}}$ solution into PVX control-plant leaves resulted in strong necrotic effects, as it did in the $M E K 1$-, $S I P K-$, and $N T F 6$-silenced plants. Infiltration of $M A P K K K \alpha-$, $M E K 2-$, and $W I P K$-silenced plants failed to lead to a reaction, even $72 \mathrm{~h}$ after the onset of elicitation (Fig. 1B). No necrotic effects were observed with the PBS treatment. The Nep $1_{\text {Mo }}$ protein with a mutated heptameric peptide motif displayed no necrosis-inducing activity and did not elicit defense responses and was no longer used in follow-up assays. These results indicated that the MAPKKK $\alpha-$ MEK2-WIPK cascade participates in signaling that leads to cell death induced by Nep1 $1_{M o}$.

\section{MAPKKK $\alpha$-MEK2-WIPK cascade regulates}

Nep1 $1_{M_{0}}$-induced $\mathrm{H}_{2} \mathrm{O}_{2}$ accumulation.

Studies focused on MAPK and reactive oxygen species (ROS) signaling in plants (Pitzschke and Hirt 2006) have suggested that cell death mediated by MAPK is associated with hydrogen peroxide $\left(\mathrm{H}_{2} \mathrm{O}_{2}\right)$ production (Ren et al. 2002). To test whether

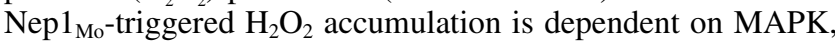
we examined $\mathrm{H}_{2} \mathrm{O}_{2}$ production by diaminobenzidine (DAB) staining. As shown in Figure $2 \mathrm{~A}$, the reddish brown-colored precipitants of oxidized DAB, an indication of $\mathrm{H}_{2} \mathrm{O}_{2}$ production, were visible in leaves from $M E K I$ - and $S I P K$-silenced and control plants treated with Nep $1_{\mathrm{Mo}}$. Light staining was observed in MAPKKK $\alpha$-, MEK2-, WIPK-, and NTF6-silenced leaves after $\mathrm{Nep}_{\mathrm{Mo}}$ treatment (Fig. 2). As with the controls, Nep $1_{\text {Mo }}$ mutant failed to trigger $\mathrm{H}_{2} \mathrm{O}_{2}$ generation, and no $\mathrm{H}_{2} \mathrm{O}_{2}$ generation was detected in silenced or control plants treated with PBS. These results suggested that the MAPKKK $\alpha-$ MEK2-WIPK cascade might include conserved signaling components that regulate $\mathrm{Nep} 1_{\mathrm{Mo}}$-activated $\mathrm{H}_{2} \mathrm{O}_{2}$ accumulation and contribute to $\mathrm{Nep} 1_{\mathrm{Mo}}$-triggered cell death.

\section{Silencing of SIPK impairs \\ Nep1 $1_{M o}$-activated stomatal closure.}

Stomata play an active role in the innate immune system (Melotto et al. 2006, 2008). It has been reported that elicitors triggered RBOH-, VPE-, and G protein-dependent stomatal closure in $N$. benthamiana (Zhang et al. 2009, 2010, 2012), but 
whether MAPK contribute to elicitor-induced stomatal closure remained unclear. Nep1 $1_{\mathrm{Mo}}$ induced stomatal closure in $M A P$ $K K K \alpha-, M E K 1-$, MEK2-, WIPK-, and NTF6-silenced plant leaves incubated with $\mathrm{Nep}_{\mathrm{Mo}}$ solution showed stomatal closure in comparison with controls. However, SIPK-silenced leaves responded weakly to this treatment, and $\mathrm{Nep} 1_{\mathrm{Mo}}$ mutant failed to induce stomatal closure. Consequently, Nep1 $1_{\mathrm{Mo}}$-induced stomatal aperture analyses were performed with all the gene-silenced plants, and SIPK-silenced plants showed a markedly reduced response to $\mathrm{Nep} 1_{\mathrm{Mo}}$ (Fig. 3). Compared with control plants, none of the gene-silenced plants showed detectable differences in stomatal phenotype after treatment with PBS. This is an indication that $S I P K$ silencing compromises $\mathrm{Nep}_{\mathrm{Mo}}$-induced stomatal closure and that not all MAPK are involved in stomatal closure.

\section{MEK2, WIPK, and SIPK are required} for NO burst induced by Nep1 $1_{\mathrm{Mo}}$.

MEK2-SIPK has been reported to mediate NO burst induced by INF1 (Asai et al. 2008; Yamamoto et al. 2004) and NO is known as a key signaling molecule of abscissic acid (ABA)-induced stomatal closure (Bright et al. 2006; Garcia-Mata and Lamattina 2002; Neill et al. 2002). In our previous study, cPTIO (a NO scavenger) restricted the $\mathrm{Nep} 1_{\mathrm{Mo}}$-induced stomatal closure, demonstrating that NO is an essential factor (Zhang et al. 2012). To investigate whether MAPK regulate NO burst, we compared NO generation in guard cells isolated from control, $M A P K K K \alpha$-, MEK1-, MEK2-, WIPK-, SIPK-, and NTF6-silenced $N$. benthamiana plants $3 \mathrm{~h}$ after $\mathrm{Nep}_{\mathrm{Mo}_{=}}$treatment. The NO generation induced by $\mathrm{Nep}_{\mathrm{Mo}}$ was slightly affected in $M A P K K K \alpha-, M E K 1-$, and NTF6-silenced plants compared with that in PVX control plants (Fig. 4A). In contrast, silencing of $M E K 2, W I P K$, and $S I P K$ markedly reduced NO genera- tion to near control levels (Fig. 4). Nep1 $1_{\text {Mo }}$ mutant failed to induce NO accumulation. These results strongly implicated the

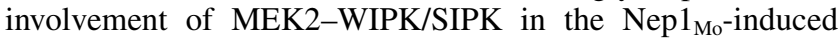
NO burst in the guard cells.

Silencing of MAPKKK $\alpha$, MEK1, MEK2, WIPK, SIPK, and NTF6 does not affect Nep1 $1_{M o}$ activation of $\mathrm{Ca}^{2+}$ generation in guard cells.

Since elicitor-treatment elevates $\left[\mathrm{Ca}^{2+}\right]_{\text {cyt }}$ profile (GarciaBrugger et al. 2006; Tavernier et al. 1995), we examined whether $\left[\mathrm{Ca}^{2+}\right]_{\text {cyt }}$ is also regulated by MAPK. The $\left[\mathrm{Ca}^{2+}\right]_{\text {cyt }}$ in the guard cells was monitored by cell-permeable Fura-2/AM. Nep $1_{\text {Mo }}$ induced a marked rise in $\left[\mathrm{Ca}^{2+}\right]_{\mathrm{cyt}}$ in the guard cells of control plants, in contrast to PBS control. Cytosolic $\mathrm{Ca}^{2+}$ fluorescence in the guard cells of both the controls and genesilenced plants was altered slightly after $\mathrm{Nep} 1_{\mathrm{Mo}}$ treatment for 3 h (Fig. 5).

\section{Silencing of $N b W R K Y 2$ interferes with Nep1 $1_{M o}$-triggered HR.}

Previous studies also indicated that the MAPK cascade participates in the cell-death signaling mediated by the WRKY transcription factor (Ekengren et al. 2003; Menke et al. 2005). We found that MAPKKKo-, MEK1-, MEK2-, WIPK-, SIPK-, and NTF6-silenced plants had decreased transcription of NbWRKY2 in response to Nep1 $1_{M o}$ (Supplementary Fig. S3). To determine whether NbWRKY2 contributes to the cell death induced by Nep $1_{\text {Mo }}$, we silenced $N b W R K Y 2$ using the PVX-VIGS system. $N b W R K Y 1$ and $N b W R K Y 2$ share $95 \%$ amino acid sequence identity and are the most abundant WRKY genes in Nicotiana species. Silencing efficiency was verified by quantitative (q)RTPCR, using unique primers to distinguish $N b W R K Y 2$ from $N b W R K Y 1$, and the results showed that $N b W R K Y 2$ transcripts

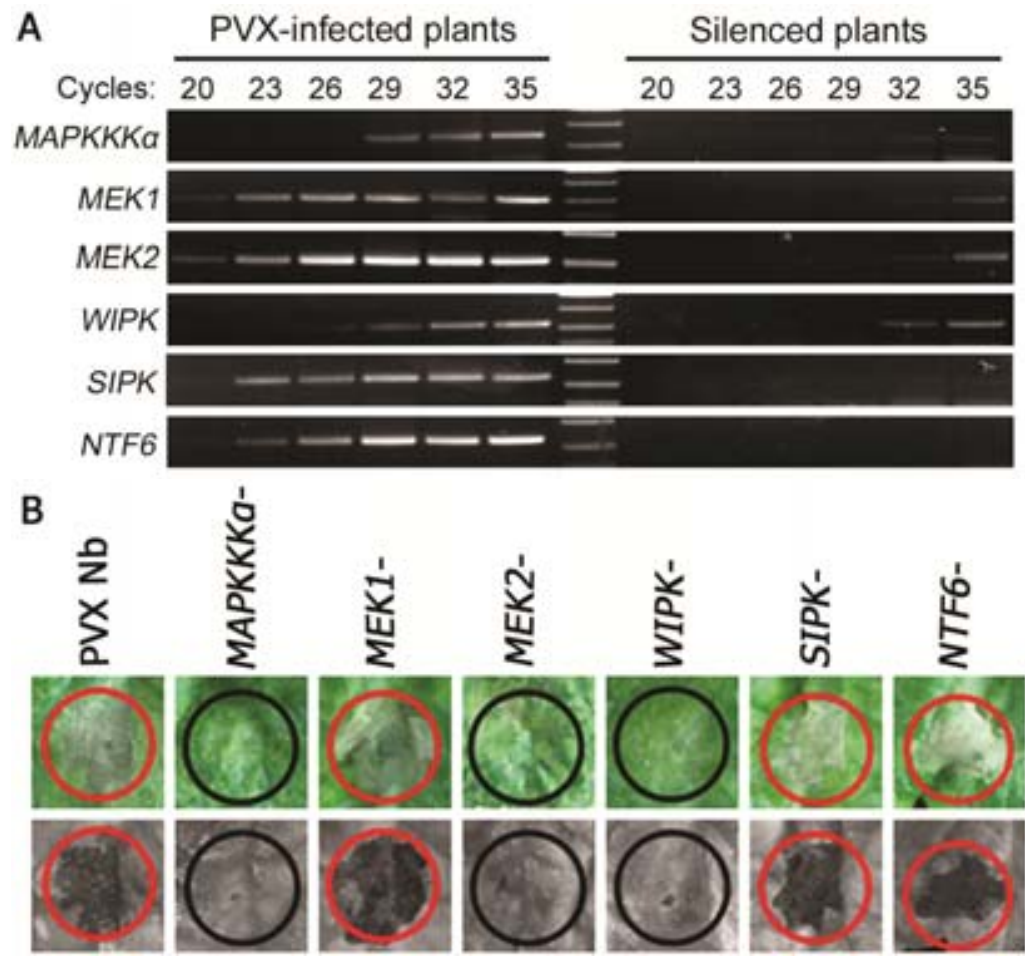

Fig. 1. Virus-induced gene silencing-mediated silencing and induction of hypersensitivity responses with Nep $1_{\text {Mo }}(50 \mathrm{nM})$. A, First-strand cDNA was generated from total RNA obtained from Potato virus X (PVX)-only plants (left panel) or from plants silenced for the genes shown on the right. Reverse transcriptionpolymerase chain reaction was performed with the cDNA and specific primers to the targeted gene and $E F 1 \alpha$ as an endogenous control. B, Leaves (representative of three replicate treatments) from control PVX, MAPKKK $\alpha$-, MEK1-, MEK2-, WIPK-, SIPK-, NTF6-silenced Nicotiana benthamiana were infiltrated with Nep1 $1_{\text {Mo }}$ solution. The red and black circles indicate cell death and no cell death, respectively. Leaves were removed from plants after 2 days of treatment (upper row) and were bleached in ethanol (lower row). 
decreased specifically in the silenced plants (Fig. 6A). Cell death induced by $\mathrm{Nep}_{\mathrm{Mo}}$ was compromised in NbWRKY2silenced plants compared with PVX control-inoculated plants (Fig. 6B). In addition, DAB staining showed light staining in NbWRKY2-silenced leaves after Nep1 $1_{\text {Mo }}$ treatment (Fig. 6C). These results suggested that NbWRKY2 functions downstream of the MAPKKK $\alpha-$ MEK2-WIPK cascade to regulate cell death induced by Nep $1_{\text {Mo }}$.

\section{NbWRKY2 functions directly downstream} of the MAPKKK $\alpha$-MEK2-WIPK cascade.

As SIPK activates WRKY through phosphorylation, it appears that SIPK and WIPK transcription factors could be functionally related (Menke et al. 2005). To test the mechanism by which MAPK and NbWRKY2 regulate $\mathrm{Nep}_{\mathrm{Mo}_{\mathrm{o}}}$-triggered $\mathrm{HCD}$, we tested the interaction between WIPK and NbWRKY2 using yeast two-hybrid assay. Only yeast hosts coexpressing WIPK and $N b W R K Y 2$ were able to grow in selective medium plus $1 \mathrm{mM}$ 3-amino-1,2,4-triazole and X-gal (Fig. 7A). Control strains expressing the strongly interacting pGADT7-RecT and pGBKT7-53 and noninteracting pGADT7-RecT and pGBKT7Lam were included as controls (Fig. 7A). To further validate this interaction, we tested the association in living cells between WIPK and NbWRKY2, using a bimolecular fluorescence complementation (BiFC) approach with WIPK fused to the C-terminus of the yellow fluorescent protein (YFP) (WIPK-YFP ${ }^{\mathrm{C}}$ ) and NbWRKY2 fused to the N-terminus of YFP (NbWRKY2$\mathrm{YFP}^{\mathrm{N}}$ ). YFP was localized to the nucleus of Arabidopsis meso- phyll protoplasts (Fig. 7B). As a negative control, WIPKYFP $^{\mathrm{C}}$ was co-transformed with the $\mathrm{YFP}^{\mathrm{N}}$ vector, and no YFP fluorescence was observed (Fig. 7B). Co-transformation of $\mathrm{NbWRKY}^{-}-\mathrm{YFP}^{\mathrm{N}}$ and the $\mathrm{YFP}^{\mathrm{C}}$ vector into protoplasts also did not result in any visible fluorescence. These results sug-

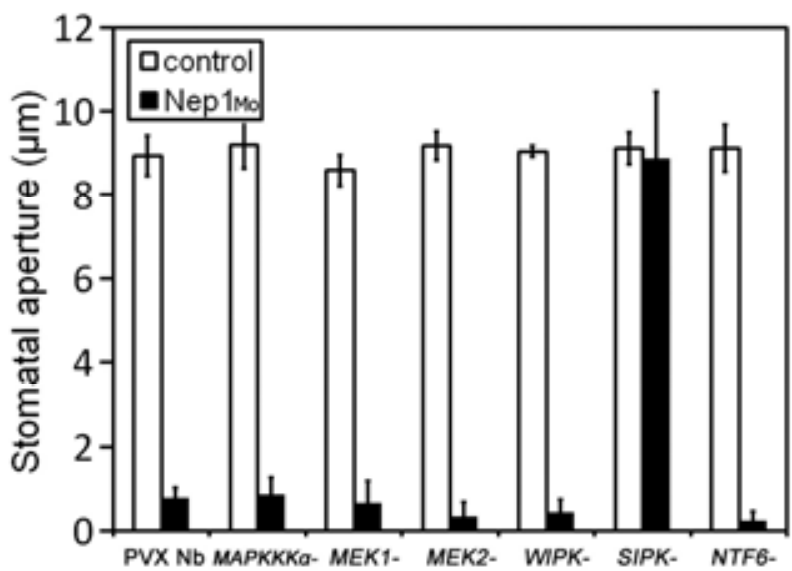

Fig. 3. Stomatal aperture measurements show that $\mathrm{Nep}_{\mathrm{Mo}}$-induced stomatal closure is reduced in SIPK-silenced Nicotiana benthamiana. Stomatal aperture was measured $3 \mathrm{~h}$ after incubation in phosphate buffered saline $(10 \mathrm{mM})$ and $\mathrm{Nep}_{\mathrm{Mo}}(50 \mathrm{nM})$. Values represent means \pm standard error from three independent experiments; $n=50$ apertures per experiment. Data were compared using the Student's $t$-test at the $95 \%$ significance level.

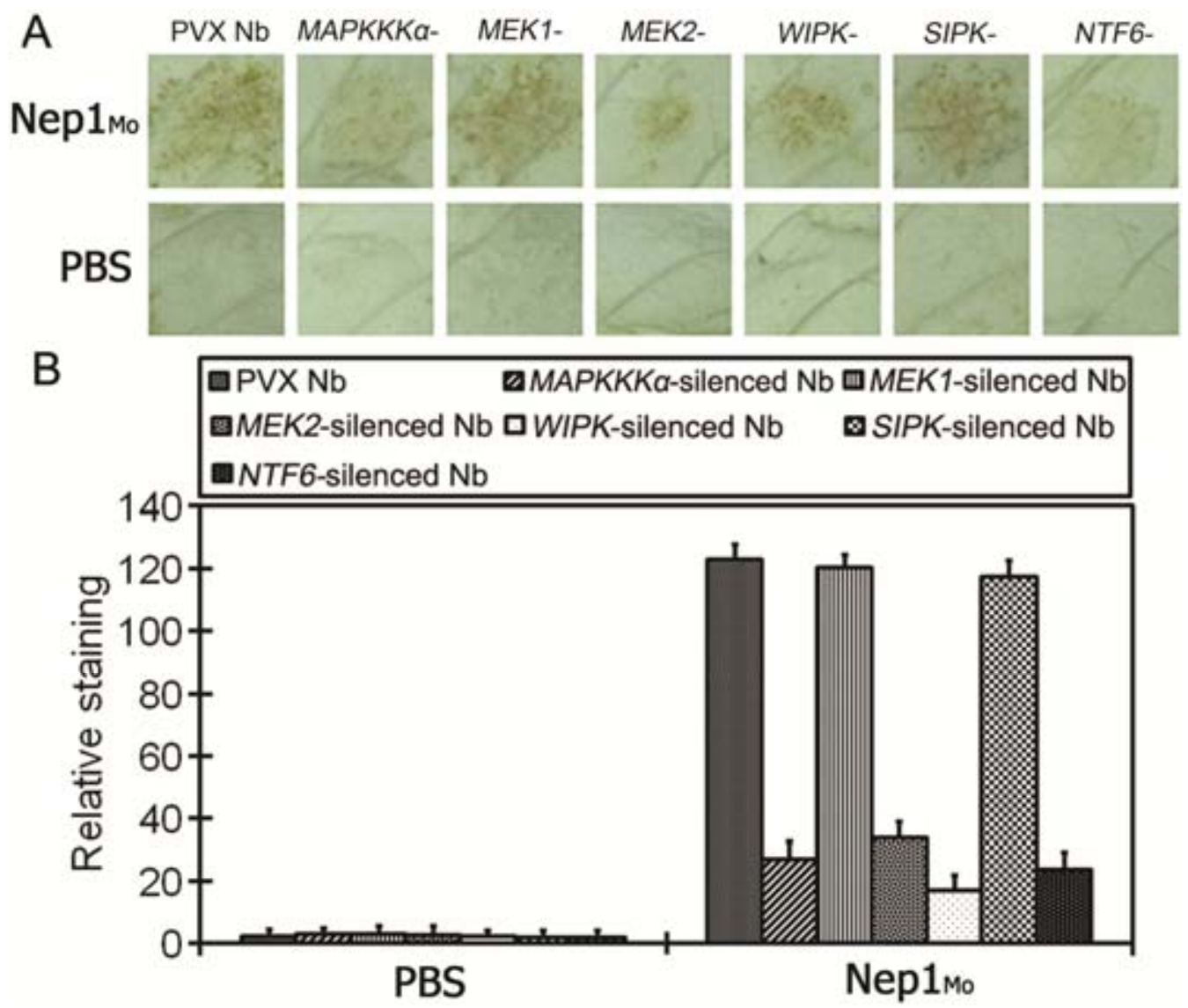

Fig. 2. In situ detection of hydrogen peroxide using diaminobenzidine staining on MAPKKK $\alpha$-, MEK1, MEK2-, WIPK-, SIPK-, and NTF6-silenced Nicotiana benthamiana leaves in response to Nep1 $1_{\text {Mo }}$. A, Nicotiana benthamiana leaves silenced for MAPKKK $\alpha, M E K 1$, MEK2, WIPK, SIPK, and NTF6 were compared with Potato virus $X(\mathrm{PVX})$-only leaves $6 \mathrm{~h}$ after infiltration of phosphate buffered saline $(10 \mathrm{mM})$ or Nep1 $1_{\mathrm{Mo}}(50 \mathrm{nM})$. Elicitation with the elicitor was conducted on plants by infiltrating an equivalent elicitor solution of $25 \mu \mathrm{l}$. B, Quantitative scoring of staining in leaves of the control and gene-silenced plants with elicitor treatment. The analysis was repeated for three sets of independently silenced plants in each experiment; the values shown were the means \pm standard deviation of duplicate assays. The experiment was repeated twice with similar results. 

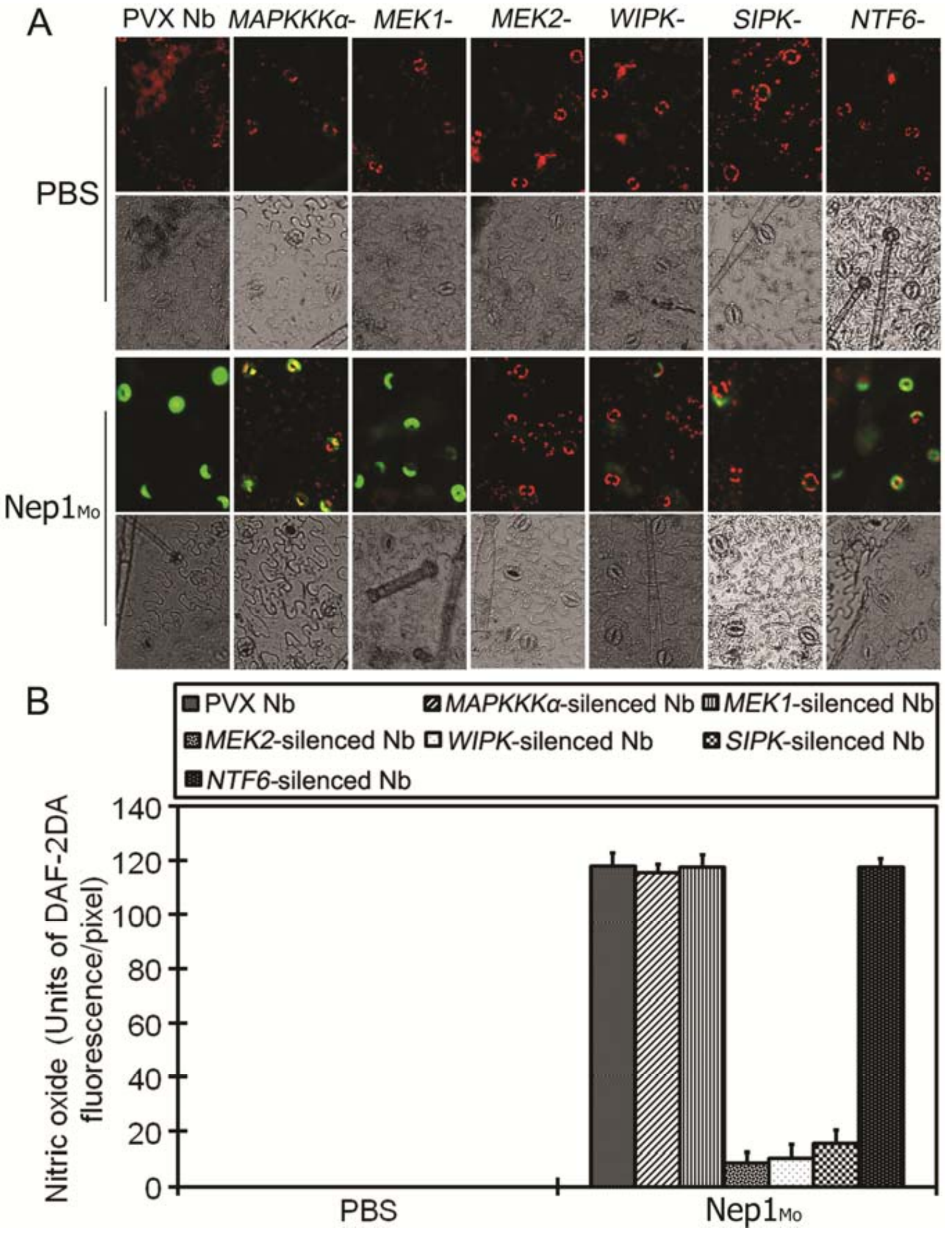

Fig. 4. Effects of silencing of MAPKKK $\alpha, M E K 1, M E K 2$, WIPK, SIPK, and NTF6 on nitric oxide (NO) burst in guard cells in response to Nep1 $1_{M o}$. A, In all cases, NO-sensitive dye 4,5-diaminofluorescein diacetate (DAF-2DA) was loaded into cells of the epidermal peels, and fluorescence was measured after addition of phosphate buffered saline $(10 \mathrm{mM})$ and $\mathrm{Nep}_{\mathrm{Mo}}(50 \mathrm{nM})$. For each treatment, fluorescence and bright field images were shown. Results from several experiments were compiled in this figure. Experiments were repeated at least three times, and representative images were shown. Green indicates NO burst. B, Quantitative analysis of in vivo NO generation monitored using DAF-2DA fluorescence. Results were presented as the mean ( $n \geq 3)$ fluorescence intensity per pixel. 
gested that WIPK interacts directly with NbWRKY2 and NbWRKY2 functions downstream of the MAPK cascade in Nep1 $1_{\mathrm{Mo}_{\mathrm{o}}}$-signaling.

\section{Nep1 $1_{M o}$ induces $N b W R K Y 2$-mediated stomatal closure and NO burst in guard cells.}

The above findings prompted us to examine the possibility that both $\mathrm{Nep} 1_{\mathrm{Mo}}$-induced stomatal closure and $\mathrm{NO}$ accumulation in the guard cells are NbWRKY2-dependent. Consistently, $N b W R K Y 2$-silenced plants significantly attenuated $\mathrm{Nep}_{\mathrm{Mo}^{-}}$ induced stomatal closure (Fig. 8A), whereas NO generation by Nep $1_{\mathrm{Mo}}$ decreased to the level induced by PBS in controls (Fig. 8B). These results indicated that, in addition to the MEK2-WIPK cascade, NbWRKY2 might also participate in the $\mathrm{Nep} 1_{\mathrm{Mo}}$-induced production of NO in the guard cells.

\section{SIPK interacts with NbWRKY2.}

The hyposensitivity to Nep1 $1_{\text {Mo }}$ promotion of stomatal closure in $S I P K$ - and NbWRKY2-silenced plants also prompted us to investigate whether SIPK physically interacts with NbWRKY2. Again, only yeast cells coexpressing SIPK and NbWRKY2 were able to grow in selective medium, while the controls did not (Fig. 9A). In BiFC, fluorescence within the nucleus can be observed in $N$. benthamiana leaves coexpressing SIPK and NbWRKY2. In contrast, no fluorescence can be detected in the leaves coexpressing NbWRKY2 and p2YC vector, the p2YN vector and SIPK, or the negative control p2YN and $\mathrm{p} 2 \mathrm{YN}$ vector (Fig. 9B). To further validate this interaction, a coimmunoprecipitation assay was performed. The ability of NbWRKY2 anti-His antibody to precipitate SIPK in the presence of recombinant NbWRKY2 protein but not in its absence (Fig. 9C) was demonstrated. The observations confirmed that SIPK is an interacting partner of NbWRKY2 in stomatal closure.

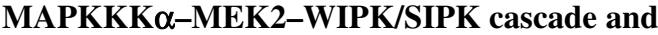 NbWRKY2 participate in Nep1 $1_{\mathrm{Mo}_{0}}$-triggered resistance of $N$. benthamiana to $P$. nicotianae.}

Since elicitors can induce systemic resistance in plants (Garcia-Brugger et al. 2006; Yang et al. 2009), we tested whether silencing of MAPKKKa, MEK2, WIPK, SIPK, and $N b W R K Y 2$ had any effects on $\mathrm{Nep}_{\mathrm{Mo}}$-triggered disease resistance. Silenced leaves were infiltrated with $10 \mu \mathrm{l}$ of $\mathrm{Nep}_{\mathrm{Mo}}$ at one spot and, $4 \mathrm{~h}$ later, the transcript level of pathogenesis-related protein (PR2b, X53600) was found approximately 30-fold higher after $\mathrm{Nep}_{\mathrm{Mo}}$ treatment in $N$. benthamiana (Supplementary Fig. S4). The leaf surfaces opposite the Nep1 $1_{\mathrm{Mo}}$-infiltrated sides were inoculated with $2 \times 2 \mathrm{~mm}$ mycelial plugs of $P$. nicotianae. Systemic resistance was determined $48 \mathrm{~h}$ after $P$. nicotianae inoculation by comparing and measuring the size of lesions. The typical water-soaked Phytophthora lesions appeared

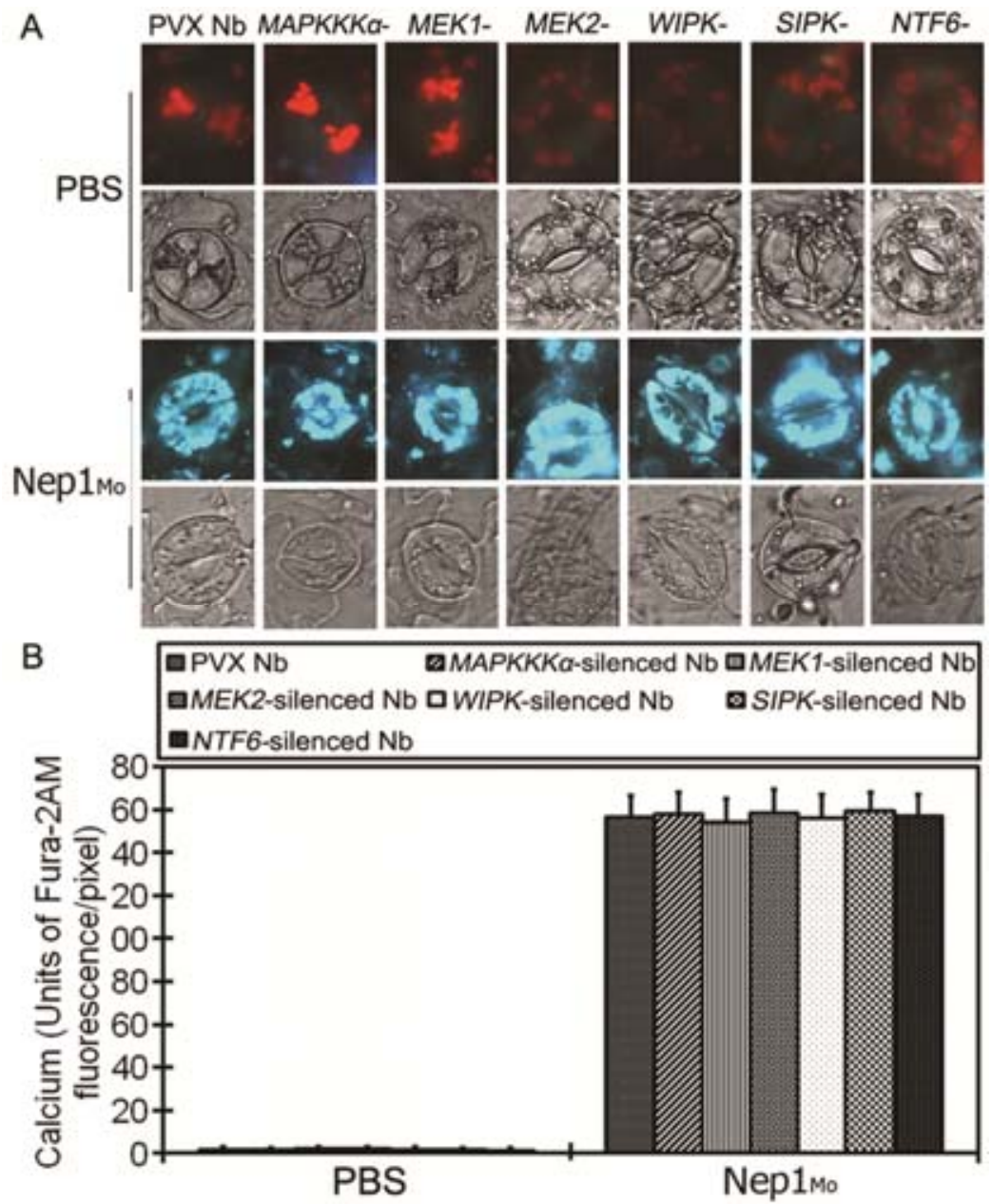

Fig. 5. Nep1 $1_{\mathrm{Mo}}$ activation of $\mathrm{Ca}^{2+}$ generation in guard cells of control and $M A P K K K \alpha-, M E K 1$-, $M E K 2-$, WIPK-, SIPK-, and NTF6-silenced plants. A, Leaf epidermal peels prepared from the control or gene-silenced plants were loaded with the $\mathrm{Ca}^{2+}$ dye Fura- 2 AM prior to incubation in phosphate buffered saline $(10 \mathrm{mM})$ and $\mathrm{Nep} 1_{\mathrm{Mo}}(50 \mathrm{nM})$. In each case, corresponding fluorescence and bright field images are shown. The areas of the peel subjected to analysis are greater than those shown in the figure. This experiment was repeated three times. Representative cells from one of three experiments are shown. In each experiment, a minimum of three epidermal peels were used as treatment replicates. B, Quantitative analysis of in vivo Ca ${ }^{2+}$ generation monitored using Fura2 AM fluorescence as shown in A. Results are presented as the mean $(n \geq 3)$ fluorescence intensity per pixel. 
within $24 \mathrm{~h}$ postinoculation, and the expanding disease lesions around the inoculated spots were observed in all silenced plants at $48 \mathrm{~h}$ postinoculation. However, Nep $1_{\mathrm{Mo}}$ treatment significantly inhibited the expansion of lesions in PVX control-inoculated leaves of $N$. benthamiana (Fig. 10). These findings suggested that MAPKKK $\alpha, M E K 2, W I P K, S I P K$, and NbWRKY2 are all required for $\mathrm{Nep} 1_{\mathrm{Mo}}$-induced systemic resistance to $P$. nicotianae.

MAPKKK $\alpha$ - MEK2-, WIPK-, SIPK-, and $N b W R K Y 2$-silenced plants show altered gene expression related to AOS homeostasis and transcription.

Compromised cell death in MAPKKK $\alpha-$, MEK2-, WIPK-, and $N b W R K Y 2$-silenced plants, hyposensitivity to Nep $1_{M o}$ promotion of stomatal closure in SIPK- and NbWRKY2-silenced plants, increased susceptibility to $P$. nicotianae in $M A P K K K \alpha$-, $M E K 2-, W I P K$-, SIPK-, and NbWRKY2-silenced plants after Nep $1_{M o}$ treatment, and altered accumulation of $\mathrm{H}_{2} \mathrm{O}_{2}$ and $\mathrm{NO}$ all suggested dysregulation of gene function is associated with defense-related redox control and transcription. To address this possibility, the kinetics of the expression of five selected genes after Nep $1_{\text {Mo }}$ infiltration was examined by qRT-PCR in genesilenced and control plants (Fig. 11). The transcript levels of acidic pathogenesis-related protein (PRla, X06930) and PR2b (X53600) showed no significant difference $(P>0.05)$ in $M A P K K K \alpha-, M E K 2-, W I P K-, S I P K-$, and $N b W R K Y 2$-silenced and control plants with PBS treatment but were three- to 33 fold lower in gene-silenced plants. The transcript level of HSR203J (AB091430), a HR marker gene, was approximately 1.7- to threefold lower after Nep $1_{\mathrm{Mo}}$ treatment in $M A P K K K \alpha-$, $M E K 2-, W I P K$-, and NbWRKY2-silenced plants than in control plants, which was coincident with necrotic lesion formation (Fig. 1B). In $M A P K K K \alpha, M E K 2-, W I P K-, S I P K-$, and
$\mathrm{NbWRKY2-silenced} \mathrm{and} \mathrm{control} \mathrm{plants,} \mathrm{the} \mathrm{expression} \mathrm{of} \mathrm{a} \mathrm{dis-}$ ease resistance protein (EDS1, AF479625) was induced by Nep $1_{\mathrm{Mo}}$ compared with PBS treatment and was down-regulated 1.5- to 4.5-fold in gene-silenced plants compared with control plants upon $\mathrm{Nep} 1_{\mathrm{Mo}}$ treatment. Compared with the control plants, nitric reductase (NR; AB245431) expression showed no obvious difference in $M A P K K K \alpha-, M E K 2-, W I P K-, S I P K-$, and $N b W R K Y 2$-silenced plants with PBS treatment (Fig. 11). It was approximately 2.8 - to 5.5 -fold lower in gene-silenced plants than in control plants after Nep $1_{\text {Mo }}$ treatment. In summary, an overlapping set of genes related to plant defense and transcription was found to show significant changes in expression in $M A P K K K \alpha-, M E K 2-$, WIPK-, SIPK-, and NbWRKY2-silenced plants compared with control plants.

\section{DISCUSSION}

We determined that the MAPK cascade and WRKY transcription factor are functionally required for $\mathrm{Nep} 1_{\mathrm{Mo}}$ signaling. Based on the phenotypes observed, we developed a model that involves three of these genes in the $\mathrm{Nep} 1_{\mathrm{Mo}}$-induced $\mathrm{HR}$ and stomatal closure (Fig. 12). First, our experiments confirmed the role of a MAPK cascade in Nep1 $1_{\mathrm{Mo}}$-induced HR. Based on previous reports (del Pozo et al., 2004), the MAPKKK $\alpha-$ MEK2-WIPK cascade most likely positively regulates HR mediated by Nep1 $1_{\text {Mo }}$. Second, as NbWRKY2 has been shown to physically interact with WIPK, we tentatively placed NbWRKY2 downstream of the MAPKKK $\alpha-M E K 2-W I P K$ cascade in regulating $\mathrm{HR}$ mediated by $\mathrm{Nep} 1_{\mathrm{Mo}}$. Our data further defined the pathways from the elicitor recognition event and provided important new tools for characterizing elicitor signaling. Third, the SIPK interacted with NbWRKY2 to control Nep1 ${ }_{\mathrm{Mo}}$-induced stomatal closure via the suppression of $\mathrm{NO}$
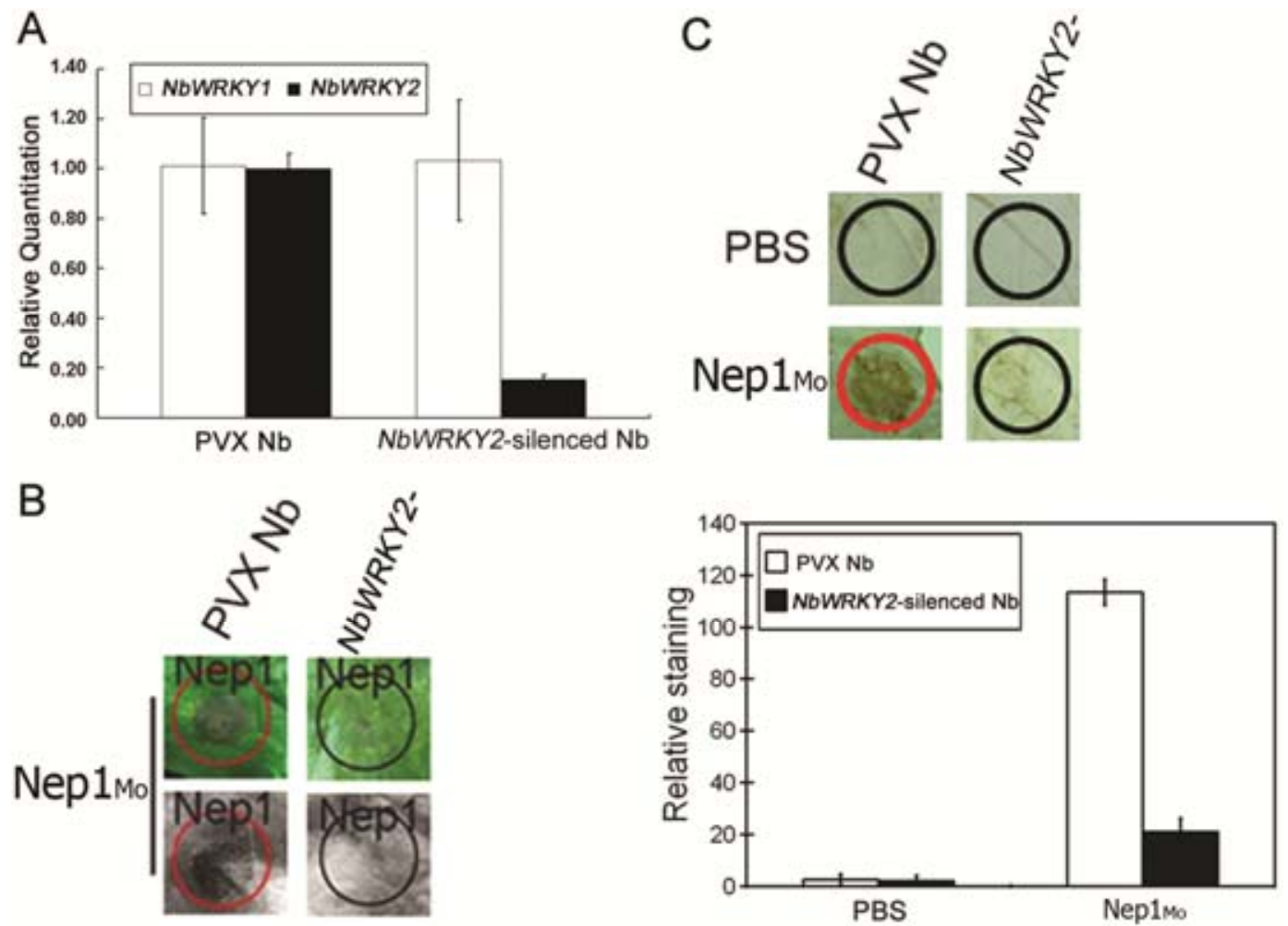

Fig. 6. $\mathrm{Nep} 1_{\mathrm{Mo}}$-mediated cell death is suppressed in Nicotiana benthamiana plants silenced for NbWRKY2. A, N. benthamiana leaves were inoculated with Potato virus $X$ (PVX)-NbWRKY2 and PVX as a control. Total RNA was isolated from leaves 3 weeks after inoculation with Agrobacterium tumefaciens containing PVX-NbWRKY2 and was used for quantitative reverse transcription-polymerase chain reaction with specific primers for $N b W R K Y 1$ and NbWRKY2. B, Inhibition of cell death on PVX-NbWRKY2 leaves compared with PVX-only leaves after infiltration of $\mathrm{Nep}_{\mathrm{Mo}}$ solution. $\mathbf{C}, \mathrm{H}_{2} \mathrm{O}_{2}$ accumulation is reduced in NbWRKY2-silenced plants compared with PVX-only plants. 


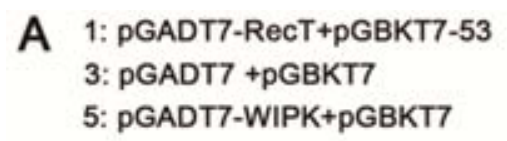
2: pGADT7-RecT+pGBKT7-Lam
4: $\mathrm{pGADT7}+\mathrm{pGBKT7-NbWRKY2}$
6: pGADT7-WIPK+pGBKT7-NbWRKY2
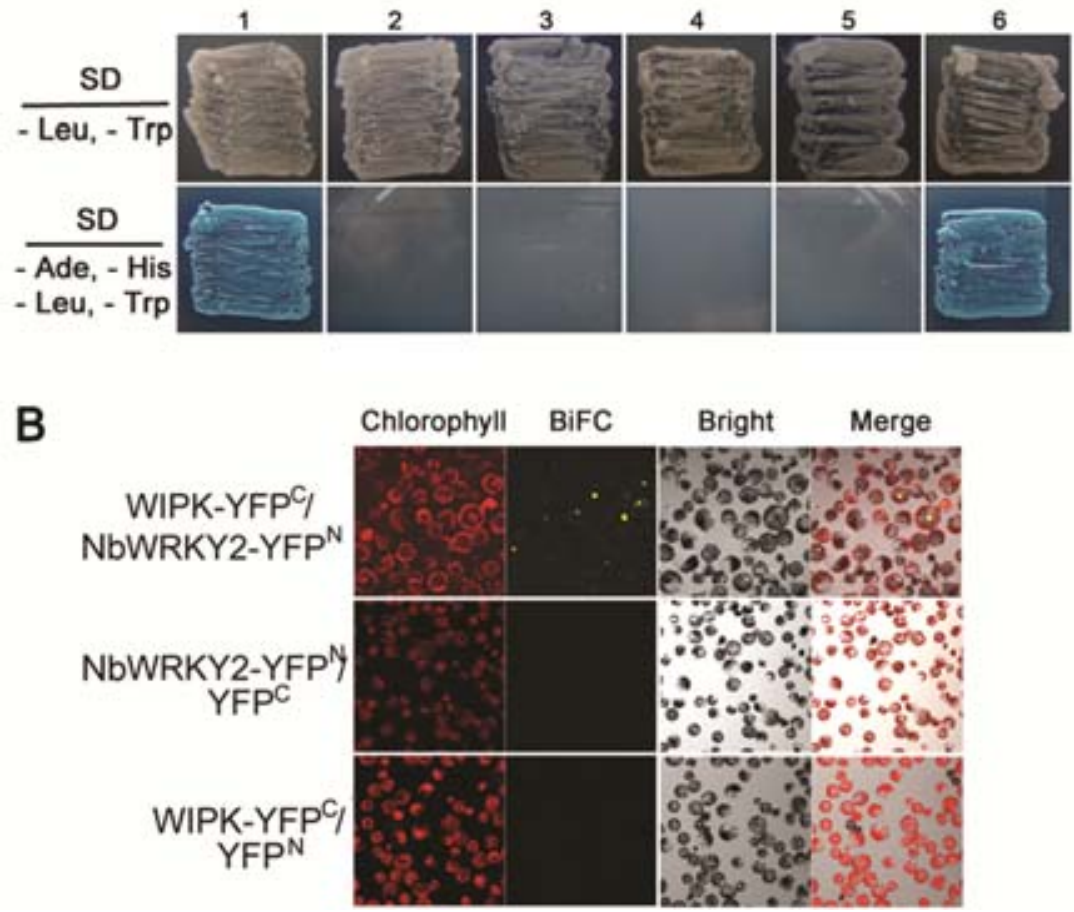

Fig. 7. WIPK and NbWRKY2 interaction. A, Yeast two-hybrid interaction of WIPK with NbWRKY2. WIPK was coexpressed with NbWRKY2 in yeast strain AH109 and was grown on synthetic dropout nutrient (SD) medium lacking Leu and Trp (upper) or SD medium lacking Ade, His, Leu, and Trp (lower). pAD-WIPK = WIPK subcloned in the prey vector pGADT7; pBD-NbWRKY2 = NbWRKY2 subcloned in the bait vector pGBKT7. The pAD-RecT+pBD53 construct served as a positive control. The pAD-RecT+pBD-Lam construct served as a negative control. B, WIPK associates with NbWRKY2 in vivo by bimolecular fluorescence complementation analysis. Arabidopsis protoplasts coexpressing WIPK and NbWRKY2 resulted in detectable yellow fluorescent protein (YFP) fluorescence in the nuclei. YFP fluorescence was excited at 488 and imaged at 518 to $540 \mathrm{~nm}$ by confocal microscopy.
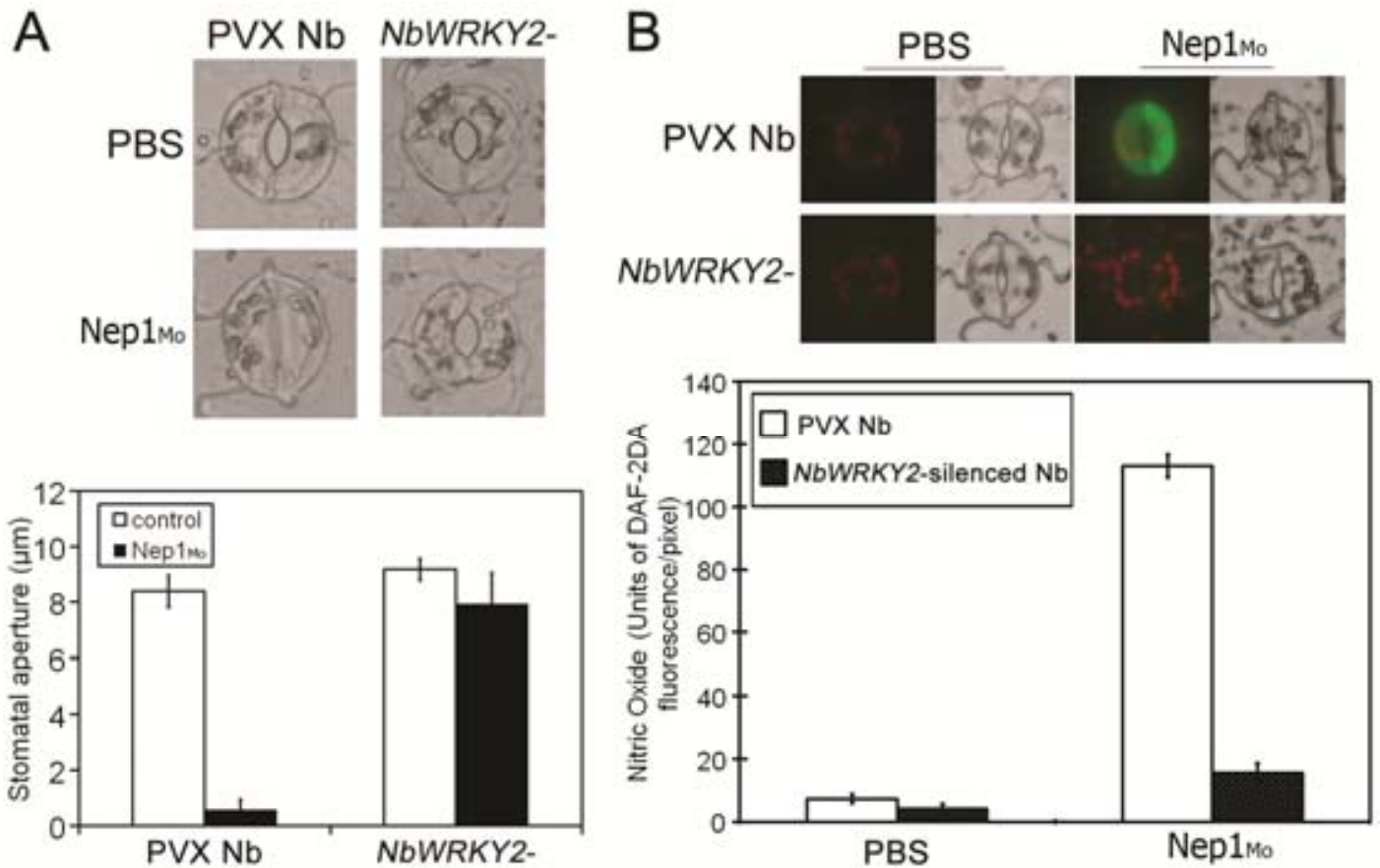

Fig. 8. NbWRKY2 participates in nitric oxide (NO)-mediated stomatal closure induced by Nep1 $1_{\mathrm{Mo}}$. A, The stomatal aperture was measured after $3 \mathrm{~h}$ of incubation in light after Nep $1_{\mathrm{Mo}}$ treatment. Values represent means \pm 1 standard error (SE) from three independent experiments; $n=50$ apertures per experiment. Data were compared using the Student's $t$-test at the 95\% significance level. B, NO-sensitive dye 4,5-diaminofluorescein diacetate was loaded into cells of the epidermal peels, and fluorescence was measured after addition of phosphate buffered saline $(10 \mathrm{mM})$ and Nep1 $1_{\mathrm{Mo}}(50 \mathrm{nM})$. Data are means $\pm \mathrm{SE}$ from three independent experiments. 
accumulation in guard cells. Our observations also suggested that the function of many defense-signaling components is conserved among different pathogen-plant interactions.

NbWRKY2 functions downstream of a MAPK cascade to positively regulate $\mathrm{Nep} 1_{\mathrm{Mo}}$-induced cell death and resistance against $P$. nicotianae.

In different interaction systems, there may be subtle (but perhaps essential) differences between PAMP-triggered immunity and cell death or immunity trigged by various elicitors. MAPK cascades are known as major pathways by which extracellular stimuli are transduced into intracellular responses in plants (Asai et al. 2002). The requirement of these kinases in defense-related signaling has been demonstrated previously in the Pto, $\mathrm{N}$-mediated, gene-for-gene interaction and in the FLS2-mediated response to flagellin (Asai et al. 2002; Zhang and Klessig 1998, 2001). Silencing of SIPK leads to hypersensitivity of the Harpinmediated HR and higher levels of ROS (which is different for Nep $1_{\text {Mo }}$ elicitation) (Samuel et al. 2005). Similarly, VIGS of $W I P K$ and SIPK genes reduced the resistance to a bacterial

\section{A 1: pGADT7-RecT + pGBKT7-53 \\ 3: pGADT7 + pGBKT7 \\ 5: pGADT7-SIPK + pGBKT7}

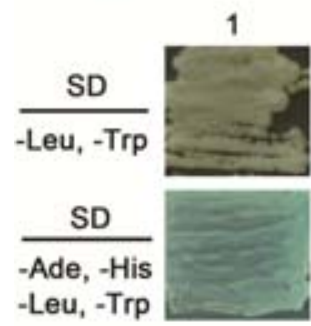

B

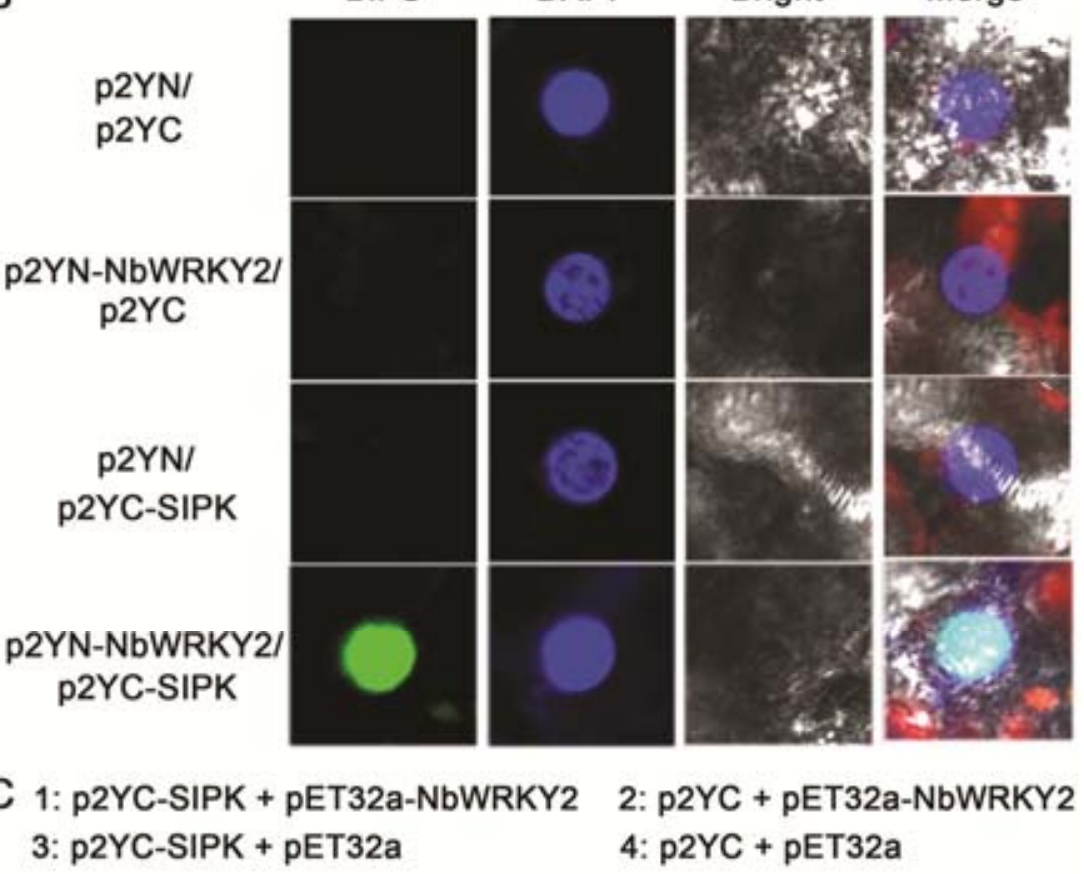

2: pGADT7-RecT + pGBKT7-Lam

4: PGADT7 + pGBKT7-NbWRKY2

6: PGADT7-SIPK + pGBKT7-NbWRKY2

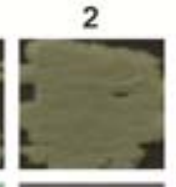

3
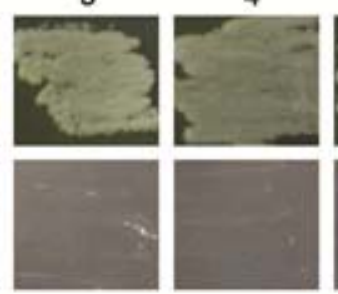

6
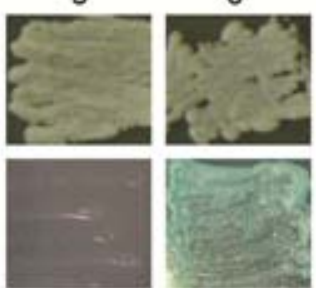
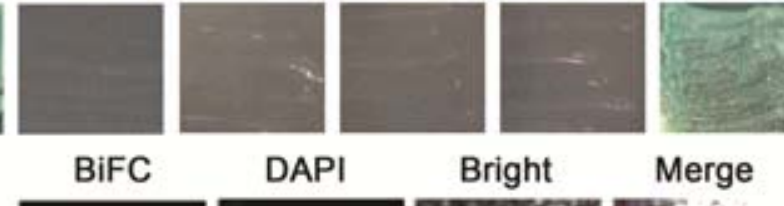

3: $\mathrm{p} 2 \mathrm{YC}-\mathrm{SIPK}+\mathrm{pET} 32 \mathrm{a}$

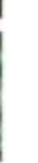

\section{(1)}

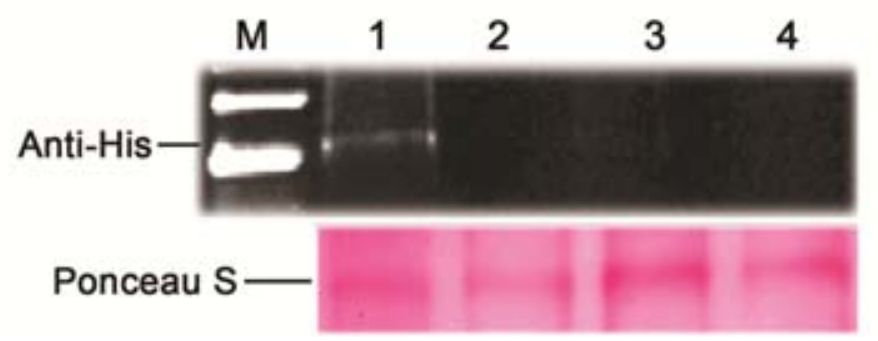

Fig. 9. SIPK and NbWRKY2 interaction. A, Yeast two-hybrid interaction of SIPK with NbWRKY2. pAD-SIPK, SIPK subcloned in the prey vector pGADT7; pBD-NbWRKY2, NbWRKY2 subcloned in the bait vector pGBKT7. The pAD-RecT+pBD-53 construct served as a positive control. The pADRecT+pBD-Lam construct served as a negative control. B, Bimolecular fluorescence complementation visualization of SIPK and NbWRKY2 interaction in agrobacterium-infiltrated Nicotiana benthamiana leaves. Confocal and bright field images of epidermal leaf cells from N. benthamiana infiltrated with a mixture of Agrobacterium suspensions harboring constructs encoding the indicated fusion proteins. C, Co-immunoprecipitation of NbWRKY2 with SIPK. NbWRKY2 or SIPK protein was incubated with NbWRKY2 anti-His antibody in the presence (lanes 1 and 2) or absence (lanes 3 and 4) of NbWRKY2, and complexes were pulled down with protein A agarose and were resolved by sodium dodecyl sulfate polyacrylamide gel electrophoresis on a $10 \%$ gel. Ponceau S staining shows the amount of input protein for NbWRKY2 and SIPK, respectively. Size markers are shown on the left of the panel. 
pathogen but had no effect on INF1-induced hypersensitive response in Nicotiana benthamiana (Sharma et al 2003). Furthermore, SIPK-suppression has differential effects on some hrpZ and $\beta$-megaspermin-induced defense responses in tobacco (Hall et al 2007). In the study, WIPK and SIPK or their orthologs were activated and were found necessary for the defense response. Notably, two entire MAPK cascades have been identified downstream of FLS2 and Pto, consisting of MEKK1, MAPKKK $\alpha$, MAPKK, MEK4/MEK5, MEK2, and MAPK, MPK3/MPK6, and WIPK, respectively (Asai et al. 2002; del Pozo et al. 2004). WIPK and SIPK are the direct substrates for MEK2 mediating N-response signaling in $N$. benthamiana (Jin et al. 2003). Here, we showed that cell death and systemic resistance against $P$. nicotianae from Nep1 $1_{\mathrm{Mo}}$ is compromised in $M A P K K K \alpha$-, MEK2-, and WIPK-silenced plants (Figs. 1 and $9)$, suggesting that $\mathrm{Nep} 1_{\mathrm{Mo}_{\mathrm{o}}}$-induced $\mathrm{PCD}$ and systemic resistance are mediated by a MAPK cascade. Our findings further reinforced previous observations that signaling events initiated by diverse pathogens converge at the point of MEK2-WIPK kinases. Additionally, $\mathrm{Nep} 1_{\mathrm{Mo}}$-induced and Pto- and $N$ genemediated cell death may share a similar HCD mechanism that depends on the MAPKKK $\alpha-$ MEK2-WIPK cascade.

NbWRKY2 was found to interact with WIPK by yeast twohybrid and BiFC analyses (Fig. 7). To date, several transcription factors, including TGA, WRKY, Myb, and EREBP, have been shown to act downstream of various MAPK (Asai et al.

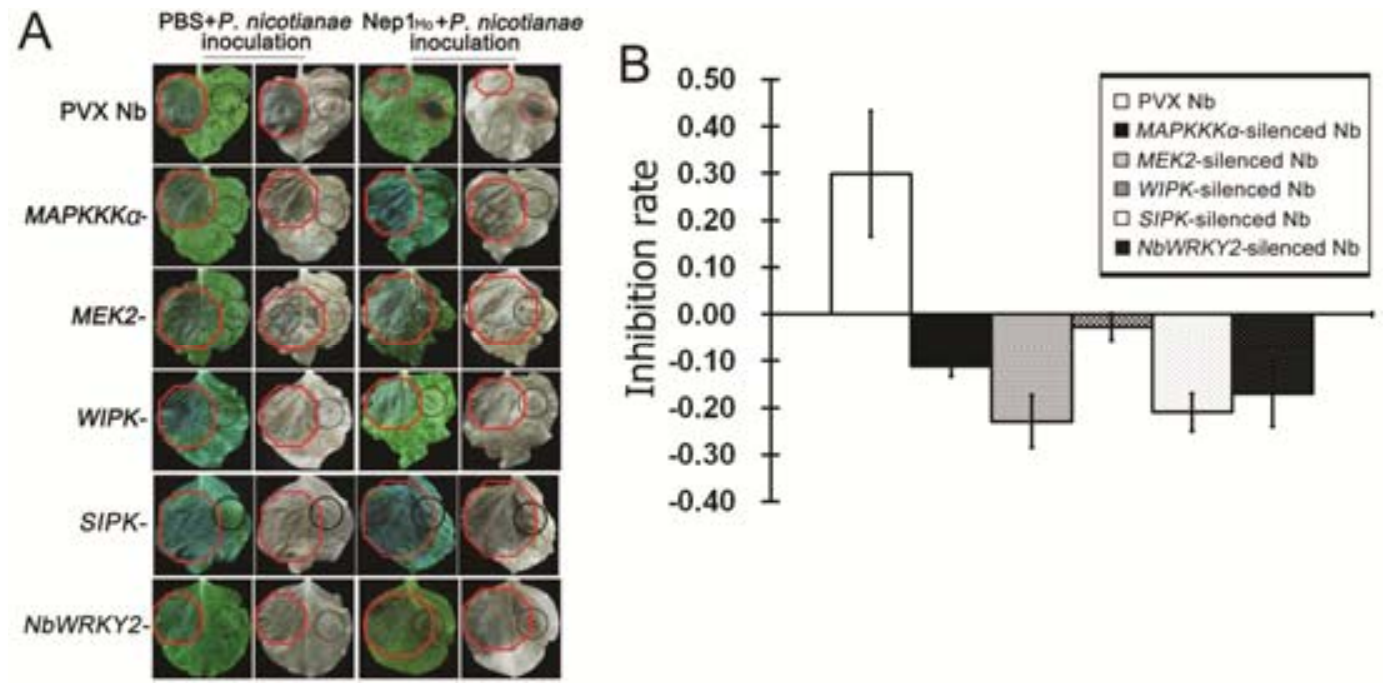

Fig. 10. MAPKKK $\alpha$-, MEK2-, WIPK-, SIPK-, and NbWRKY2-silenced plants display enhanced sensitivity to Phytophthora nicotianae. A, For the control and gene-silenced plants, fully expanded leaves collected $4 \mathrm{~h}$ after Nep1 ${ }_{\mathrm{Mo}}$ treatment $(10 \mu \mathrm{l}$, black circle) were inoculated with a $2 \times 2 \mathrm{~mm}$ hyphal plug on the left leaf surfaces opposite the Nep1 $1_{\mathrm{Mo}}$-infiltrated sides. Then, the leaves were placed in petri dishes containing filter paper saturated with sterilized distilled water and were kept under a 16-h-day and 8-h-night regime at $25^{\circ} \mathrm{C}$. Pictures of the lesions were taken at $48 \mathrm{~h}$ postinoculation and the lesion diameter (red circle) was measured. B, Resistance evaluation based on diameter of lesion spots. The inhibition rate $=$ (diameter of necrosis with phosphate buffered saline treatment - diameter of necrosis with $\mathrm{Nep}_{\mathrm{Mo}}$ treatment)/diameter of control. Data are means \pm standard error from eight experiments.
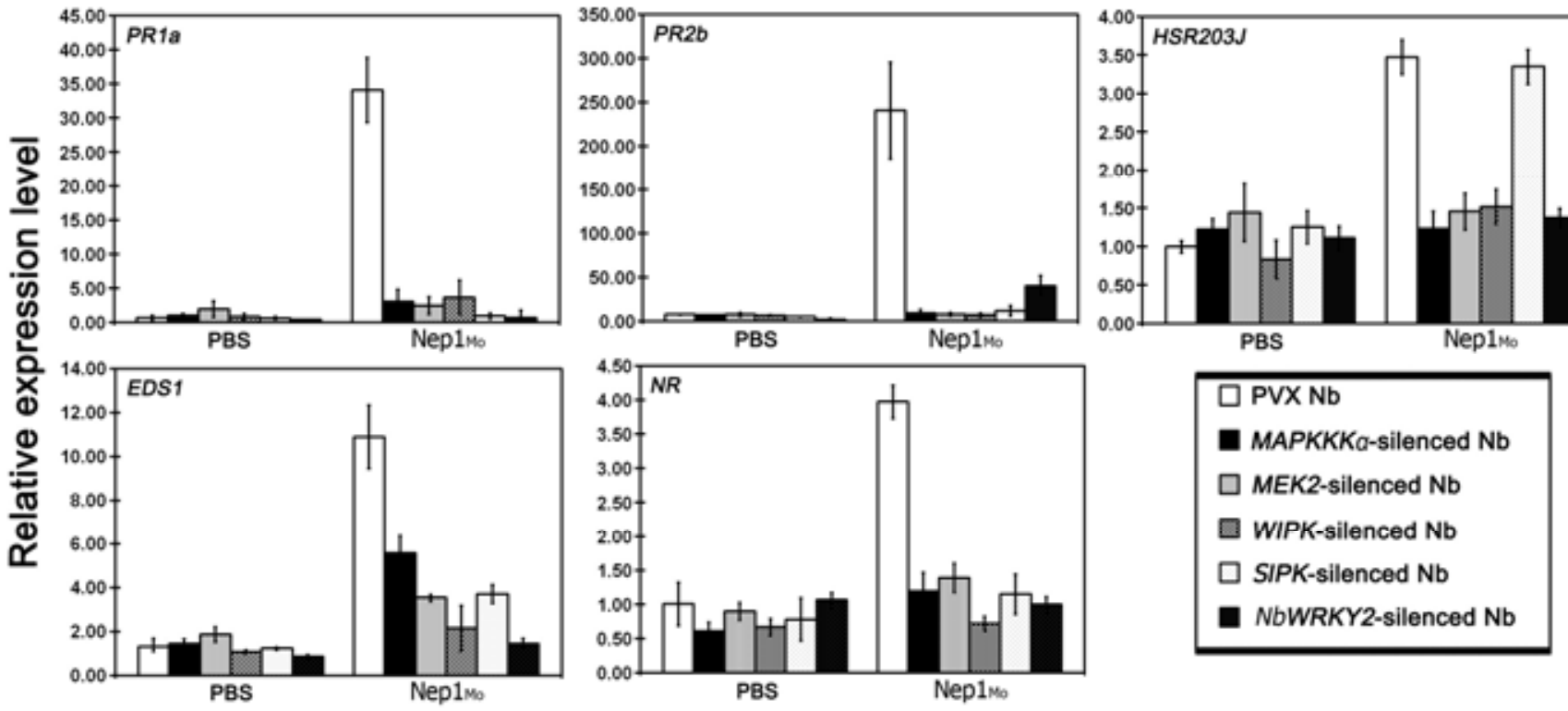

Fig. 11. Expression analysis of genes associated with redox control and transcription in $M A P K K K \alpha$-, MEK2-, WIPK-, SIPK-, and NbWRKY2-silenced plants by quantitative reverse transcription-polymerase chain reaction. At $6 \mathrm{~h}$ after treatment with or without Nep1 $1_{\mathrm{Mo}}(50 \mathrm{nM})$, leaf samples were harvested from the inoculation site, the lower, and the upper leaves; $N$. benthamiana $E F-1 \alpha$ expression is used to normalize the expression value in each sample, and relative expression values were determined against buffer or Potato virus $X$ (PVX)-infected plants using the comparative cycle threshold method $\left(2^{-\Delta \Delta C t}\right)$. Bars represent mean (three biological replicates) \pm standard deviation. 
2002; Cheong et al. 2003; Ekengren et al. 2003; Kim and Zhang 2004; Liu et al. 2004). Here, the increased susceptibility to $P$. nicotianae and compromised cell death observed in NbWRKY2silenced plants might also indicate that NbWRKY2 plays an important role in $\mathrm{Nep}_{\mathrm{Mo}}$-mediated HR. We found that the silencing of $M A P K K K \alpha, M E K 2, W I P K$, and $N b W R K Y 2$ results in compromised cell death and resistance against $P$. nicotianae, suggesting that NbWRKY2 acts downstream of the MAP$\mathrm{KKK} \alpha-\mathrm{MEK} 2-\mathrm{WIPK}$ cascade to participate in plant defense in $N$. benthamiana. This also shows that non-race specific resistance mechanisms are also compromised in MAPK cascade genes and $N b W R K Y 2$-silenced plants.

\section{Silencing of components}

in the MAPKKK $\alpha-M E K 2-W I P K$ cascade compromises

Nep1 $\mathrm{Mo}_{0}$-induced $\mathrm{H}_{2} \mathrm{O}_{2}$ accumulation.

We used VIGS in N. benthamiana to define biological functions for MAP kinase genes in $\mathrm{H}_{2} \mathrm{O}_{2}$ accumulation during defense signal-specific elicitation in response to $\mathrm{Nep} 1_{\mathrm{Mo}}$ (Fig. 2). The possibility exists that elicitor activation of MAPK in plants is one of the mechanisms that control $\mathrm{H}_{2} \mathrm{O}_{2}$ generation, perhaps leading to cell death. Nep1 $1_{\text {Mo }}$ elicitation of Arabidopsis MAPK has been reported (Bae et al. 2006). Yoshioka and associates (2003) showed that the MEK2 pathway may be a part of an amplification cascade upstream of the NADPH oxidase genes, which are necessary for producing $\mathrm{H}_{2} \mathrm{O}_{2}$ in response to fungal infection. Here, we determined that $M A P K K K \alpha$, $M E K 2, N T F 6$, and WIPK have roles in $\mathrm{Nep}_{\mathrm{Mo}_{\mathrm{o}}}$-induced $\mathrm{H}_{2} \mathrm{O}_{2}$ accumulation, indicating that activation of the MAPKKK $\alpha-$ MEK2-WIPK cascade might be required for the $\mathrm{H}_{2} \mathrm{O}_{2}$ burst leading to $\mathrm{HR}$ cell death induced by $\mathrm{Nep} 1_{\mathrm{Mo}}$. On the other hand, NTF6-silenced plants showed decreased DAB-staining intensity and normal cell death in response to Nep1 $1_{\mathrm{Mo}}$, indicating no strict correlation exists between $\mathrm{H}_{2} \mathrm{O}_{2}$ production and cell death. For example, although AtrbohD, a plant NADPH
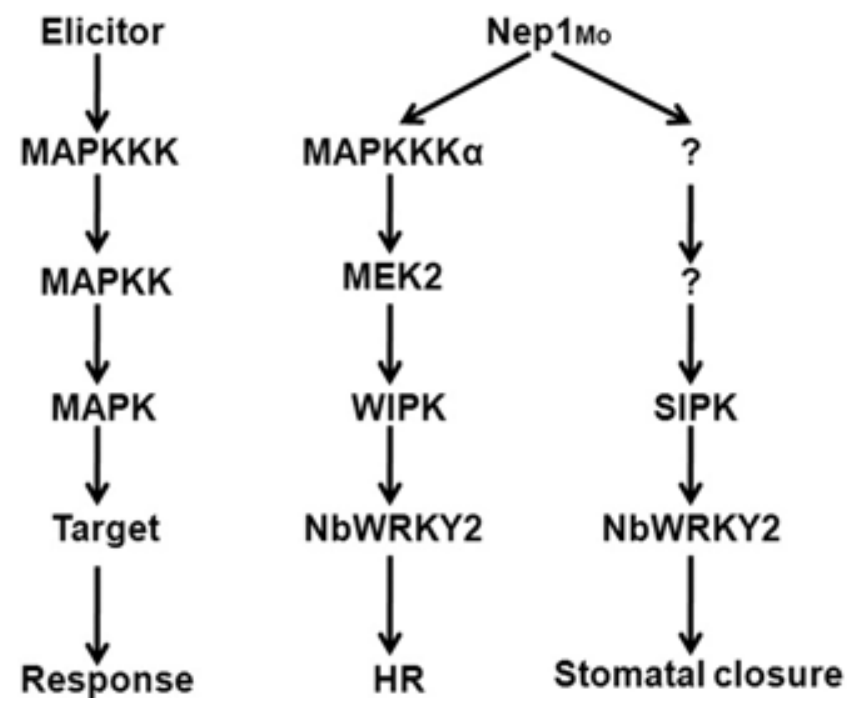

Fig. 12. Speculative model of new signaling components in the $\mathrm{Nep} 1_{\mathrm{Mo}^{-}}$ activated signaling pathway. Virus-induced gene silencing of various candidate genes in Nicotiana benthamiana placed $M A P K K K \alpha, M E K 2, W I P K$, $S I P K$, and $N b W R K Y 2$ downstream of $N e p 1_{M o}$ recognition event. Question marks (?) indicate positions of an unknown but hypothesized component acting between Nep1 $1_{\text {Mo }}$ and the MAPK cascade, the position of a putative MAPKKK, or MAPKK. NbWRKY2 interacts physically with WIPK to regulate the MAPKKK $\alpha-M E K 2-W I P K$ cascade-mediated hypersensitive response $(\mathrm{HR})$ induced by $\mathrm{Nep} 1_{\mathrm{Mo}}$; on the other hand, SIPK and NbWRKY2 contribute to Nep1 $1_{\mathrm{Mo}}$-induced stomatal closure. Silencing of each of the signaling genes involved in HR and stomatal closure compromises $\mathrm{Nep} 1_{\mathrm{Mo}}$-induced resistance. oxidase, contributes to $\mathrm{H}_{2} \mathrm{O}_{2}$ accumulation to a greater degree than AtrbohF, plant cell death was more severely inhibited in an AtrbohF mutant than in an AtrbohD mutant after treatment with avirulent Pseudomonas syringae DC3000 expressing the AvrRpm1 elicitor (Torres et al. 2002). Similarly, in cryptogeintreated tobacco plants, $\mathrm{H}_{2} \mathrm{O}_{2}$ plays an essential role in plant cell death by provoking AOS-mediated lipid peroxidation in the light (Montillet et al. 2005) but not in the dark (Rustérucci et al. 1999).

\section{SIPK- and NbWRKY2-silenced plants show a reduced stomatal closure response}

to $\mathrm{Nep1}_{\mathrm{Mo}}$ and resistance against $P$. nicotianae.

Our results showed that silencing of SIPK and NbWRKY2 compromised $\mathrm{Nep}_{\mathrm{Mo}}$-induced stomatal closure that was accompanied by less NO accumulation in the guard cells. Furthermore, NbWRKY2 interacted with SIPK in yeast two-hybrid and BiFC analyses (Figs. 3, 4, 8, and 9). This suggests that SIPK interacts with NbWRKY2 to control Nep1 $1_{\mathrm{Mo}}$-mediated stomatal closure. $\mathrm{NO}$ is a key mediator of ABA-induced stomatal closure in peas (Neill et al. 2002), Vicia faba (GarciaMata and Lamattina 2002), and Arabidopsis (Bright et al. 2006). These data indicated that SIPK and NbWRKY2 mediate $\mathrm{Nep} 1_{\mathrm{Mo}_{\mathrm{o}}}$-triggered stomatal closure via NO signaling.

An increasing number of studies in plants have strongly confirmed the importance of stoma function in plant immunity, and stomatal closure observed is an output of PTI. Our results showed the induced resistance against $P$. nicotianae from $\mathrm{Nep}_{\mathrm{Mo}}$ was also compromised in SIPK- and NbWRKY2silenced plants (Fig. 9), suggesting that SIPK and NbWRKY2 are key regulators of plant immunity to regulate stomatal apertures, inhibiting the entry of pathogens into the plant leaf during infection.

In this study, silencing of MEK2, WIPK, SIPK, and $N b W R K Y 2$ was found to suppress NO accumulation in the guard cells triggered by $\mathrm{Nep} 1_{\mathrm{Mo}}$; however, only $S I P K$ and

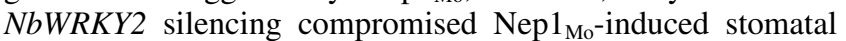
closure. This result suggested that stomatal movement is associated with NO accumulation, but reduced NO accumulation may not result in inhibition of stomatal closure. NO, serving as a signaling molecule, not only regulates plant physiological processes, but also coordinates the HR and plant innate immunity (Ali et al. 2007; Dangl 1998).

Cross talk between MAPK cascades, $\mathrm{Ca}^{2+}, \mathrm{H}_{2} \mathrm{O}_{2}$, and NO.

$\mathrm{NO}, \mathrm{H}_{2} \mathrm{O}_{2}$, and calcium are essential signaling components during cell death induced not only by elicitors but also by biotic and abiotic stress. The present study highlights that NO accumulation by a fungal elicitor such as Nep $1_{\mathrm{Mo}}$ is also mediated by the MAPK. Other studies have also shown that the MEK2-SIPK/NTF4 cascade controls the INF1-mediated NO burst (Asai et al. 2008). Our results support the theory that common signaling components such as $\mathrm{NO}, \mathrm{H}_{2} \mathrm{O}_{2}$, and $\mathrm{Ca}^{2+}$ participate in the transduction of diverse signals emanating from biotic or abiotic stress, including UV-B or ozone stress (da Silva et al. 2011; Fujita et al. 2006; Holley et al. 2003).

Nep $1_{\text {Mo }}$ raises the levels of $\mathrm{H}_{2} \mathrm{O}_{2}$ and calcium in guard cells. The marked enhancement in the levels of both $\mathrm{H}_{2} \mathrm{O}_{2}$ and $\mathrm{Ca}^{2+}$ by $\mathrm{Nep} 1_{\mathrm{Mo}}$ emphasizes that $\mathrm{Nep} 1_{\mathrm{Mo}_{0}}$-mediated cell death requires both $\mathrm{H}_{2} \mathrm{O}_{2}$ and calcium. The participation of both $\mathrm{H}_{2} \mathrm{O}_{2}$ and $\mathrm{Ca}^{2+}$ has also been observed in elicitor signaling in other systems (Zhang et al. 2009, 2010). No visible differences of $\mathrm{Ca}^{2+}$ fluorescence imaging in guard cells were detected between the control and MAPKKK $\alpha$-, MEK1-, MEK2-, WIPK-, SIPK-, NTF6-silenced plants $3 \mathrm{~h}$ after elicitor treatment, which is consistent with the finding that $\mathrm{Ca}^{2+}$ spiking is an early event induced by elicitors and acts upstream of MAPK (Garcia-Brugger 
et al. 2006). Using pharmacological experiments, apoplastic $\mathrm{Ca}^{2+}$ influx was demonstrated to be important in the oxidative burst, and $\mathrm{Ca}^{2+}$ was shown to activate an AOS in vitro in tobacco and tomato (Miura et al. 1995, 1999; Sagi and Fluhr 2001). In this study, we found that silencing of SIPK inhibited Nep $1_{M o}$-induced NO accumulation in guard cells, which concurs with previous findings that SIPK is required for the NO burst induced by INF1 (Asai et al. 2008). The MEK2WIPK/SIPK cascade regulates the NO burst triggered by Nep1 $1_{\mathrm{Mo}}$ as well as HR cell death, which suggests that a balance of NO and AOS determines the fate of plants.

Nep1 $1_{M o}$ alters gene expression targeting signal transduction.

We found that silencing of $N b W R K Y 2$ in $N$. benthamiana blocked cell death induced by $\mathrm{Nep} 1_{\mathrm{Mo}}$ (Fig. 6), and WIPK was able to physically interact with NbWRKY2 transcription factors (Fig. 7). Therefore, we tentatively place NbWRKY2 downstream of WIPK. To date, several transcription factors, including WRKY family members, have been shown to act downstream of various MAPK (Liu et al. 2004). A study demonstrated that WRKY1 also interacts with SIPK in the hypersensitive response-like cell death in tobacco (Menke et al. 2005). This interaction occurs because activated SIPK binds to the $\mathrm{W}$ box sequence (Menke et al. 2005).

Nep $1_{\text {Mo }}$ triggered a complex immune response in Arabidopsis, including MAPK activation, production of NO, ethylene, camalexin, and callose, and extensive reprogramming of the transcriptome (Motteram et al. 2009; Qutob et al. 2006). qRTPCR analyses showed that the transcript expression of PRla, $P R 2 b$, and $E D S 1$, which are associated with resistance signaling and plant defense execution, is up-regulated by $\mathrm{Nep} 1_{\mathrm{Mo}}$ and is down-regulated in gene-silenced plants upon Nep1 $1_{\text {Mo }}$ treatment. These results suggested that the genes encoding putative disease resistance proteins are involved in $\mathrm{Nep} 1_{\mathrm{Mo}}$-induced cell death and plant defense.

NO can be synthesized in plants by nitrite reduction with nitrate reductase, oxidation of arginine to citrulline by napoline synthase, and a nonenzymatic NO generation system (Crawford 2006). Here, qRT-PCR analyses showed that silencing of $M A P K K K \alpha, M E K 2, W I P K$, and $N b W R K Y 2$ appears to decrease the expression of $N R$ after Nep1 $1_{\text {Mo }}$ treatment. The decreased NO accumulation in $M E K 2-, W I P K$-, and SIPK-silenced plants indicates that NR may participate in $\mathrm{Nep} 1_{\mathrm{Mo}_{0}}$-induced NO production. Further characterization of $\mathrm{Nep}_{\mathrm{Mo}}$-mediated cell death signaling in plants will enhance our understanding of biological processes that lead to susceptibility in plant-pathogen interactions and improve our ability to control plant diseases.

\section{Nep1 $1_{M o}$ triggers plant defense.}

In this study, we comprehensively characterized the immune response to Nep $1_{\text {Mo }}$. Results from this study and previous work allowed us to conclude that $\mathrm{Nep} 1_{\mathrm{Mo}}$ is responsible for a complex immune response. This response includes MAPK activation, production of $\mathrm{NO}, \mathrm{Ca}^{2+}$, and $\mathrm{H}_{2} \mathrm{O}_{2}$, extensive reprogramming of the transcriptome, and plant resistance. Cell death and tissue necrosis terminate this massive defense response. The effects of $\mathrm{Nep} 1_{\mathrm{Mo}}$ resemble those triggered by interaction of AvrPto and Pto, suggesting that both signals are perceived as equivalent determinants of microbial nonself recognition by the plant and trigger similar activation of the plant surveillance system. These findings also facilitate the study of the molecular architecture of merging signaling pathways and possible synergisms between them in one plant. In comparison to the activation of Pto-mediated plant cultivar-specific resistance, relatively little is known about the signaling pathways mediating non-cultivar specific plant resistance. Our isolation of fungiderived $\mathrm{Nep}_{\mathrm{Mo}}$ and characterization of its defense-inducing potential in $N$. benthamiana now provide suitable tools for such an approach, to determine its relationship with toxin signaling and PAMP signaling.

Furthermore, regarding the presence of Nep1-encoding genes in the genomes of both monocot-infecting fungal pathogens and dicot-infecting plant pathogens, determining whether Nep1triggered plant pathogen interaction from different systems shares the same downstream factors or whether diversified components have evolved will also be interesting.

\section{MATERIALS AND METHODS}

\section{Plant materials, elicitors, and treatment protocols.}

$N$. benthamiana plants were grown in a growth chamber under a 16-h-light and 8-h-dark cycle at $25^{\circ} \mathrm{C}$. A needleless syringe was used to inject $25 \mu \mathrm{l}$ of Magnaporthe oryzae $\mathrm{Nep}_{\mathrm{Mo}}$ $(50 \mathrm{nM})$ into tiny cuts on the underside of the leaf, thereby flooding the apoplastic space. To prepare Nep $1_{\mathrm{Mo}}$, overnight cultures of Escherichia coli BL21 cells carrying pET32b harboring the Nepl $1_{M o}$ gene (GenBank accession number MGG_08454) were diluted (1:100) in Luria-Bertani medium containing ampicillin $(50 \mu \mathrm{g} / \mathrm{ml})$ and were incubated at $37^{\circ} \mathrm{C}$. When the optical density at $600 \mathrm{~nm}$ of the culture reached 0.6 , Nep $1_{M o}$ secretion into the culture medium was induced via the addition of $0.4 \mathrm{mM}$ isopropyl-beta-D-thiogalactopyranoside for $6 \mathrm{~h}$. Comparison of NLP primary sequences found in public databases revealed strong conservation of a central hepta-peptide motif, GHRHDWE (Ottmann et al. 2009). The site-directed mutations within the GHRHDWE site of Nep1 $1_{\text {Mo }}$, located at amino acids 123 to 129 , were constructed using a two-step amplification procedure to create a Nep $1_{\mathrm{Mo}}$ mutant 1 and a mutant 2 by two pairs of primers (F1: GAATTCATGCTTCCCAAGT TCTTCACTCTCC, R1: ACCTCCACCTCCACCTCCACCC ATGCCCGGCGACGGCGAGTC; F2: GGTGGAGGTGGAG GTGGAGGTAACGTCATCATCTGG, R2: AAGCTTTCATT GGAAAACCTTGGCCAGGTTG) and then, GHRHDWE was individually exchanged for glycine by overlap PCR. Nep $1_{\mathrm{Mo}}$ and $\mathrm{Nep} 1_{\mathrm{Mo}}$ mutant were expressed as a His tag fusion protein. Proteins were examined by sodium dodecyl sulfate polyacrylamide gel electrophoresis (SDS-PAGE). Protein purification was performed with the Ni-nitrilotriacetic acid resin (Qiagen, Valencia, CA, U.S.A.), and the purified proteins were dialyzed against a phosphate-buffered saline (PBS) buffer ( $\mathrm{pH} 7.4)$ and were stored at $-20^{\circ} \mathrm{C}$ prior to use. Protein concentrations were determined using the Bradford reagent (Qutob et al. 2006), and concentrated stock solutions $(500 \mathrm{nM})$ were prepared.

\section{DNA constructs and seedling infection for virus-induced gene silencing.}

VIGS for the MAPKKK $\alpha$ (GenBank accession number AY500155), MEK1 (GenBank accession number AJ302651), MEK2 (GenBank accession number AF325168), WIPK (GenBank accession number AB098729), SIPK (GenBank accession number AB098730), NTF6 (GenBank accession number AY547494), and NbWRKY2 (GenBank accession number TC12974) genes in $N$. benthamiana was performed using potato virus $X$ (PVX), as previously described by Zhang and associates $(2009,2010,2011)$. The MAPKKK $\alpha, M E K 1$, MEK2, WIPK, SIPK, NTF6, and NbWRKY2 inserts were 276, $257,267,262,262,274$, and $323 \mathrm{bp}$, respectively, which were derived from the $3^{\prime}$ termini of their respective open reading frames and were inserted into the PVX vector separately in the antisense direction to generate PVX-MAPKKK $\alpha$, PVXMEK1, PVX-MEK2, PVX-WIPK, PVX-SIPK, PVX-NTF6, and PVX-NbWRKY2. The constructs containing the inserts were transformed into Agrobacterium tumefaciens GV3101. Bacterial suspensions were applied to the undersides of $N$. 
benthamiana leaves, using a 1-ml needleless syringe. Plants exhibited mild mosaic symptoms 3 weeks after inoculation. The third or fourth leaf above the inoculated leaf in which silencing was most consistently established was used for further analyses.

\section{DAB staining.}

Following the methods of Gan and associates (2009), leaves were harvested $6 \mathrm{~h}$ after Nep $1_{\mathrm{Mo}}$ treatment and were immediately vacuum-infiltrated for 20 min with PBS ( $\mathrm{pH}$ 7.4) containing $0.5 \%$ (wt/vol) DAB. The leaves were placed in light for $10 \mathrm{~h}$ and were then boiled for $20 \mathrm{~min}$ in $80 \%$ ethanol. The intensity and pattern of DAB staining was assessed visually. Quantitative scoring of $\mathrm{H}_{2} \mathrm{O}_{2}$ staining in leaves was analyzed using Quantity One software (Bio-Rad, Segrate, Italy).

RNA isolation, semiquantitative RT-PCR, and qRT-PCR.

As described in Zhang and associates (2010), real-time RTPCR was performed using the cDNA and gene-specific primers (Supplementary Table 1). Each cDNA was amplified by quantitative PCR using SYBR PrimeScript RT-PCR kit (TaKaRa, Dalian, China) and the ABI 7300 real-time PCR system (Applied Biosystems, Foster City, CA, U.S.A.). To ensure that similar amounts of cDNA were used for silenced and nonsilenced plants, we ran parallel reactions with elongation factor $1 \alpha$ $(E F 1 \alpha)$ primers as controls. $N$. benthamiana $E F$ - $\alpha$ expression was used to normalize the expression value in each sample, and relative expression values were determined against buffer or PVX-infected plants using the comparative cycle threshold method $\left(2^{-\Delta \Delta \mathrm{Ct}}\right)$ (Zhang et al. 2010).

\section{Stomatal aperture measurements.}

Stomatal apertures were measured as described by Chen and associates (2004) and Zhang and associates (2009, 2010). Leaves were derived from various plants silenced for $M A P$ KKK $\alpha, M E K 1, M E K 2$, WIPK, SIPK, NTF6, NbWRKY2, and for controls. After a 3-week inoculation, samples were harvested from the third and fourth leaves above the inoculation site. Abaxial (lower) epidermis was peeled off and floated in 5 $\mathrm{mM} \mathrm{KCl}, 50 \mathrm{mM} \mathrm{CaCl}_{2}$, and $10 \mathrm{mM}$ MES-Tris (pH 6.15) in light for at least $2 \mathrm{~h}$ to open the stomata fully before experimentation, to minimize the effects of other factors in stomatal response, because the mesophyll signals can also significantly influence stomatal behavior. The epidermal strips were then followed by $\mathrm{Nep} 1_{\mathrm{Mo}}$ treatment for $3 \mathrm{~h}$ to induce a stomatal response. The maximum diameter of stomata was measured under an optical microscope. At least 50 apertures in each treatment were obtained. The experiments were repeated three times.

\section{NO measurement in guard cells.}

NO accumulation was determined using the fluorophore 4,5-diaminofluorescein diacetate (DAF-2DA, Sigma-Aldrich, St. Louis) according to Ali and associates (2007). Epidermal strips were prepared from control and gene-silenced plants and were incubated in $5 \mathrm{mM} \mathrm{KCl}$ and $10 \mathrm{mM}$ MES-Tris (pH 6.15) in light for $2 \mathrm{~h}$, followed by incubation in $20 \mu \mathrm{M}$ DAF-2DA for $1 \mathrm{~h}$ in the dark at $25^{\circ} \mathrm{C}$, and finally, were rinsed three times with $10 \mathrm{mM}$ Tris- $\mathrm{HCl}(\mathrm{pH}$ 7.4) to wash off excess fluorophore. Images of guard cells were obtained $3 \mathrm{~h}$ after $\mathrm{Nep} 1_{\mathrm{Mo}}$ treatment under a fluorescence microscope (excitation wavelength, $470 \mathrm{~nm}$; emission wavelength, $515 \mathrm{~nm}$; Leica DMR, Wetzlar, Germany). Fluorescence emission from guard cells was analyzed using Quantity One software.

\section{Measurement of $\mathrm{Ca}^{2+}$ in guard cells.}

Following Chen and associates (2004), the epidermal strips were peeled gently and incubated in $5 \mathrm{mM}$ fura-2/acetoxyl- methyl ester (Dojin Biochemicals, Kumamoto, Japan) for 60 min at $37^{\circ} \mathrm{C}$ in darkness. After aspiration of the fura-2/acetoxylmethyl ester solution, the glass slides were rinsed. Guard cell images were taken $3 \mathrm{~h}$ after Nep $1_{\mathrm{Mo}}$ treatment by a fluorescence microscope and were analyzed with the software Quantity One.

\section{Yeast two-hybrid and BiFC assays.}

The yeast two-hybrid was carried out in an AH109 yeast host as described previously (James et al. 1996). WIPK, SIPK, and $N b W R K Y 2$ were amplified by RT-PCR from N. benthamiana total cDNA. The $N b W R K Y 2$ gene was cloned into pGBKT7 as bait, and the WIPK and SIPK genes were cloned into pGADT7 as prey, respectively. Yeast transformation was performed by the LiAc and polyethylene glycol method, as described in the Yeast Protocols Handbook (Clontech, Palo Alto, CA, U.S.A.). Yeast transformants were screened on synthetic dropout nutrient medium with galactose and $\mathrm{X}$-gal but lacking uracil, histidine, and tryptophan. Blue colonies indicate a positive interaction between two proteins in yeast.

\section{Co-immunoprecipitation.}

For co-immunoprecipitation experiments, NbWRKY2 was subcloned in pET32a, was expressed in E. coli BL21, and was purified by standard nickel-affinity chromatography (Novagen, Madison, WI, U.S.A.). SIPK was cloned to $\mathrm{p} 2 \mathrm{YC}$, and the construct was transiently expressed through agrobacteriummediated transformation into $N$. benthamiana. Protein extracts were prepared by grinding $400 \mathrm{mg}$ of leaf tissue in $1 \mathrm{ml}$ RIPA lysis and extraction buffer (Beyotime, Jiangsu, China) in the presence of $0.1 \mathrm{mM}$ phenylmethylsulfonyl fluoride (Beyotime). Purified NbWRKY2, SIPK protein, and $2 \mu \mathrm{l}$ of antihemagglutinin antibody were co-incubated overnight at $4^{\circ} \mathrm{C}$. Proteins from the sample lysate were fractionated using SDSPAGE, were transferred to an Immobilon-PSQ polyvinylidene diuoride membrane, and were then blocked using PBS $(\mathrm{pH}$ 7.4). Mouse anti-His monoclonal antibodies (Sigma-Aldrich) were added at a ratio of 1:5,000 and were incubated at room temperature for $90 \mathrm{~min}$. The membrane was then incubated with a goat anti-mouse IRDye 800CW (Odyssey, Lincoln, NE, U.S.A.), were processed, and signals were detected using ODYSSEY LI-COR, with excitation set at 780 and $800 \mathrm{~nm}$.

\section{Disease resistance assay.}

A Phytophthora parasitica var. nicotianae strain (Pp016) was maintained on LBA medium (60 $\mathrm{g}$ of lima bean, $20 \mathrm{~g}$ of

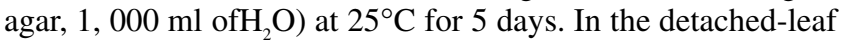
inoculation assay, fully expanded leaves collected $4 \mathrm{~h}$ after Nep $1_{\text {Mo }}$ treatment $(10 \mu \mathrm{l})$ were inoculated with a $2 \times 2 \mathrm{~mm}$ hyphal plug on the left leaf surfaces opposite the Nep1 $1_{\mathrm{Mo}}$-infiltrated sides. Then the leaves were placed in petri dishes containing filter paper saturated with sterilized distilled water and were kept under a 16 -h-day and 8 -h-night regime at $25^{\circ} \mathrm{C}$. Pictures of the lesions were taken at $48 \mathrm{~h}$ postinoculation and the lesion diameter was measured. The inhibition rate $=($ diameter of necrosis with PBS treatment - diameter of necrosis with Nep $1_{\text {Mo }}$ treatment)/diameter of control.

\section{ACKNOWLEDGMENTS}

This work was supported in part by the National Natural Science Foundation of China (grant numbers 30871605 and 31071645), the Fundamental Research Funds for the Central Universities (KYZ201105), and the Project of Jiangsu of China (grant number Sx(2009)54, X. B. Zheng). We thank D. Baulcombe (Sainsbury Laboratory, John Innes Centre, Norwich, U.K.) for the gift of PVX vector and Agrobacterium strains, X. Zhou (College of Agriculture and Biotechnology, Zhejiang University, Hangzhou, China) for the gift of Nicotiana benthamiana seeds. We also thank P. Wang of Louisiana State University Health Sciences Center, New Orleans, 
LA, U.S.A. for helpful comments and M. Jiang (College of Life Science, Nanjing Agricultural University, Nanjing, China) for the gift of SIPK and WIPK antibodies.

\section{LITERATURE CITED}

Ali, R., Ma, W., Lemtiri-Chlieh, F., Tsaltas, D., Leng, Q., von Bodman, S., and Berkowitz, G. A. 2007. Death don't have no mercy and neither does calcium: Arabidopsis cyclic nucleotide gated channel 2 and innate immunity. Plant Cell 19:1081-1095.

Amsellem, Z., Cohen, B. A., and Gressel, J. 2002. Engineering hypervirulence in a mycoherbicidal fungus for efficient weed control. Nat Biotechnol 20:1035-1039.

Andreasson, E., Jenkins, T., Brodersen, P., Thorgrimsen, S., Petersen, N. H., Zhu, S., Qiu, J. L., Micheelsen, P., Rocher, A., Petersen, M., Newman, M. A., Nielsen, H. B., Hirt, H., Somssich, I., Mattsson, O., and Mundy, J. 2005. The MAP kinase substrate MKS1 is a regulator of plant defense responses. EMBO (Eur. Mol. Biol. Organ.) J. 24:2579-2589.

Asai, S., Ohta, K., and Yoshioka, H. 2008. MAPK signaling regulates nitric oxide and NADPH oxidase-dependent oxidative bursts in Nicotiana benthamiana. Plant Cell 20:1390-1406.

Asai, T., Tena, G., Plotnikova, J., Willmann, M. R., Chiu, W. L., GomezGomez, L., Boller, T., Ausubel, F. M., and Sheen, J. 2002. MAP kinase signalling cascade in Arabidopsis innate immunity. Nature 415:977983

Bae, H., Kim, M., Sicher, R., Bae, H. J., and Bailey, B. 2006. Necrosisand ethylene-inducing peptide from Fusarium oxysporum induces a complex cascade of transcripts associated with signal transduction and cell death in Arabidopsis. Plant Physiol. 141:1056-1067.

Bright, J., Desikan, R., Hancock, J. T., Weir, I. S., and Neill, S. J. 2006. ABA-induced NO generation and stomatal closure in Arabidopsis are dependent on $\mathrm{H}_{2} \mathrm{O}_{2}$ synthesis. Plant J. 45:113-122.

Cechin, A. L., Sinigaglia, M., Lemke, N., Echeverrigaray, S., Cabrera, O. G., Pereira, G. A., and Mombach, J. C. M. 2008. Cupin: A candidate molecular structure for the Nep1-like protein family. BMC Plant Biol. 8:50.

Chen, R. E., and Thorner, J. 2007. Function and regulation in MAPK signaling pathways: Lessons learned from the yeast Saccharomyces cerevisiae. Biochim. Biophys. Acta. 1773:1311-1340.

Chen, Y. L., Huang, R. F., Xiao, Y. M., Lu, P., Chen, J., and Wang, X. C. 2004. Extracellular calmodulin-induced stomatal closure is mediated by heterotrimeric $\mathrm{G}$ protein and $\mathrm{H}_{2} \mathrm{O}_{2}$. Plant Physiol. 136:4096-4103.

Cheong, Y. H., Moon, B. C., Kim, J. K., Kim, C. Y., Kim, M. C., Kim, I. H., Park, C. Y., Kim, J. C., Park, B. O., Koo, S. C., Yoon, H. W., Chung, W. S., Lim, C. O., Lee, S. Y., and Cho, M. J. 2003. BWMK1, a rice mitogen-activated protein kinase, locates in the nucleus and mediates pathogenesis-related gene expression by activation of a transcription factor. Plant Physiol. 132:1961-1972.

Crawford, N. M. 2006. Mechanisms for nitric oxide synthesis in plants. J. Exp. Bot. 57:471-478.

da Silva, L. F., Dias, C. V., Cidade, L. C., Mendes, J. S., Pirovani, C. P., Alvim, F. C., Pereira, G. A. G., Aragão, F. J. L., Cascardo, J. C. M., Costa, M. G. C. 2011. Expression of an oxalate decarboxylase impairs the necrotic effect induced by Nep1-like protein (NLP) of Moniliophthora perniciosa in transgenic tobacco. Mol. Plant Microbe Interact. 24:839-848

Dangl, J. L. 1998. Plants just say NO to pathogens. Nature 394: 585-588.

Dangl, J. L., Dietrich, R. A., and Richberg, M. H. 1996. Death don't have no mercy: Cell death programs in plant-microbe interactions. Plant Cell 8:1793-1807.

del Pozo, O., Pedley, K. F., and Martin, G. B. 2004. MAPKKK $\alpha$ is a positive regulator of cell death associated with both plant immunity and disease. EMBO (Eur. Mol. Biol. Organ.) J. 23:3072-3082.

Ekengren, S. K., Liu, Y., Schiff, M., Dinesh-Kumar, S. P., and Martin, G. B. 2003. Two MAPK cascades, NPR1, and TGA transcription factors play a role in Pto-mediated disease resistance in tomato. Plant J. 36:905-917.

Eulgem, T., and Somssich, I. E. 2007. Networks of WRKY transcription factors in defense signaling. Curr. Opin. Plant Biol. 10:366-371.

Fujita, M., Fujita, Y., Noutoshi, Y., Takahashi, F., Narusaka, Y., YamaguchiShinozaki, K., and Shinozaki, K. 2006. Crosstalk between abiotic and biotic stress responses: A current view from the points of convergence in the stress signaling networks. Curr. Opin. Plant Biol. 9:436-442.

Gan, Y. Z., Zhang, L. S., Zhang, Z. G., Dong, S. M., Li, J., Wang, Y. C., and Zheng, X. B. 2009. The LCB2 subunit of the sphingolipid biosynthesis enzyme serine palmitoyltransferase can function as an attenuator of the hypersensitive response and Bax-induced cell death. New Phytol. 181:127-146

Garcia-Brugger, A., Lamotte, O., Vandelle, E., Bourque, S., David, L.,
Benoit, P., Lecourieux, D., Poinssot, B., Wendehenne, D., and Pugin, A. 2006. Early signaling events induced by elicitors of plant defenses. Mol. Plant Microbe Interact. 19:711-724.

Garcia-Mata, C., and Lamattina, L. 2002. Nitric oxide and abscisic acid cross talk in guard cells. Plant Physiol. 128:790-792.

Gijzen, M., and Nürnberger, T. 2006. Nep1-like proteins from plant pathogens: Recruitment and diversification of the NPP1 domain across taxa. Phytochemistry 67:1800-1807.

Greenberg, J. T., and Yao, N. 2004. The role and regulation of programmed cell death in plant-pathogen interactions. Cell Microbiol. 6:201-211.

Hall, H. C., Samuel, M. A., and Ellis, B. E. 2007. SIPK conditions transcriptional responses unique to either bacterial or oomycete elicitation in tobacco. Mol Plant Pathol. 8:581-594.

Hammond-Kosack, K. E., and Jones, J. D. G. 1996. Resistance gene-dependent plant defense responses. Plant Cell 8:1773-1791.

Holley, S. R., Yalamanchili, R. D., Moura, D. S., Ryan, C. A., and Stratmann, J. W. 2003. Convergence of signaling pathways induced by systemin, oligosaccharide elicitors, and ultraviolet-B radiation at the level of mitogen-activated protein kinases in Lycopersicon peruvianum suspension-cultured cells. Plant Physiol. 132:1728-1738.

James, P., Halladay, J., and Craig, E. A. 1996. Genomic libraries and a host strain designed for highly efficient two-hybrid selection in yeast. Genetics 144:1425-1436.

Jin, H., Axtell, M. J., Dahlbeck, D., Ekwanna, O., Zhang, S., Staskawicz, B., and Baker, B. 2002. NPK1, an MEKK1-like mitogen-activated protein kinase kinase kinase, regulates innate immunity and development in plants. Dev. Cell 3:291-297.

Jin, H., Liu, Y., Yang, K. Y., Kim, C. Y., Baker, B., and Zhang, S. 2003. Function of a mitogen-activated protein kinase pathway in $N$ gene mediated resistance in tobacco. Plant J. 33:719-731.

Katou, S., Yamamoto, A., Yoshioka, H., Kawakita, K., and Doke, N. 2003. Functional analysis of potato mitogen-activated protein kinase kinase, StMEK1. J. Gen. Plant Pathol. 69:161-168.

Kim, C. Y., and Zhang, S. 2004. Activation of a mitogen-activated protein kinase cascade induces WRKY family of transcription factors and defense genes in tobacco. Plant J. 38:142-151.

Lam, E., Kato, N., and Lawton, M. 2001. Programmed cell death, mitochondria and the plant hypersensitive response. Nature 411:848-853.

Liu, J. L., Fiil, B. K., Petersen, K., Nielsen, H. B., Botanga, C. J., Thorgrimsen, S., Palma, K., Suarez-Rodriguez, M. C., Sandbech-Clausen, S., Lichota, J., Brodersen, P., Grasser, K. D., Mattsson, O., Glazebrook, J., Mundy, J., and Petersen. M. 2008. Arabidopsis MAP kinase 4 regulates gene expression through transcription factor release in the nucleus. EMBO (Eur. Mol. Biol. Organ.) J. 27:2214-2221.

Liu, Y., Jin, H., Yang, K. Y., Kim, C. Y., Baker, B., and Zhang, S. 2003. Interaction between two mitogen-activated protein kinases during tobacco defense signaling. Plant J. 34:149-160.

Liu, Y., Schiff, M., and Dinesh-Kumar, S. P. 2004. Involvement of MEK1 MAPKK, NTF6 MAPK, WRKY/MYB transcription factors, COI1 and CTR1 in N-mediated resistance to tobacco mosaic virus. Plant J. 38:800-809.

Ludwig, A. A., Saitoh, H., Felix, G., Freymark, G., Miersch, O., Wasternack, C., Boller, T., Jones, J. D. G., and Romeis, T. 2005. Ethylene-mediated cross-talk between calcium-dependent protein kinase and MAPK signaling controls stress responses in plants. Proc. Natl. Acad. Sci. U.S.A. 102:10736-10741.

Melotto, M., Underwood, W., Koczan, J., Nomura, K., He, S. Y. 2006. Plant stomata function in innate immunity against bacterial invasion. Cell 126:969-980.

Melotto, M., Underwood, W., and He, S. Y. 2008. Role of stomata in plant innate immunity and foliar bacterial diseases. Annu. Rev. Phytopathol. 46:101-122.

Menke, F. L. H., Kang, H.-G., Chen, Z., Park, J. M., Kumar, D., and Klessig, D. F. 2005. Tobacco transcription factor WRKY1 is phosphorylated by the MAP kinase SIPK and mediates HR-Like cell death in tobacco. Mol. Plant Microbe Interact. 18:1027-1034.

Miura, Y., Yoshioka, H., and Doke, N. 1995. An autophotographic determination of the active oxygen generation in potato tuber discs during hypersensitive response to fungal infection or elicitor. Plant Sci. 105:45-52.

Miura, Y., Yoshioka, H., Park, H. J., Kawakita, K., and Doke, N. 1999. Plasma membrane perturbation in association with calcium ion movement followed by fungal elicitor-stimulated oxidative burst and defense gene activation in potato tuber. Ann. Phytopathol. Soc. Japan 65:447-453.

Montillet, J. L., Chamnongpol, S., Rustérucci, C., Dat, J., Van De Cotte, B., Agnel, J. P., Battesti, C., Inzé, D., Van Breusegem, F., and Trianphylidès, C. 2005. Fatty acid hydroperoxides and $\mathrm{H}_{2} \mathrm{O}_{2}$ in the execution of hypersensitive cell death in tobacco leaves. Plant Physiol. 138:1516-1526.

Motteram, J., Küfner, I., Deller, S., Brunner, F., Hammond-Kosack, K. E., Nürnberger, T., Rudd, J. J. 2009. Molecular characterization and func- 
tional analysis of $M g N L P$, the sole NPP1 domain-containing protein, from the fungal wheat leaf pathogen Mycosphaerella graminicola. $\mathrm{Mol}$ Plant Microbe Interact. 22:790-799.

Mur, L. A., Kenton, P., Lloyd, A. J., Ougham, H., and Prats, E. 2008. The hypersensitive response: The centenary is upon us but how much do we know? J. Exp. Bot. 59:501-520.

Nakagami, H., Pitzschke, A., and Hirt, H. 2005. Emerging MAP kinase pathways in plant stress signalling. Trends Plant Sci. 10:339-346.

Neill, S. J., Desikan, R., Clarke, A., and Hancock, J. T. 2002. Nitric oxide is a novel component of abscisic acid signaling in stomatal guard cells. Plant Physiol. 128:13-16.

Nürnberger, T., Brunner, F., Kemmerling, B., and Piater, L. 2004. Innate immunity in plants and animals: Striking similarities and obvious differences. Immunol. Rev. 198:249-266.

Oh, C. S., Pedley, K. F., and Martin, G. B. 2009. Tomato 14-3-3 protein 7 positively regulates immunity-associated programmed cell death by enhancing protein abundance and signaling ability of MAPKKK $\alpha$. Plant Cell 22:260-272.

Ottmann, C., Luberacki, B., Küfner, I., Koch, W., Brunner, F., Weyand, M., Mattinen, L., Pirhonen, M., Anderluh, G., Seitz, H. U., Nürnberger, T., Oecking, C. 2009. A common toxin fold mediates microbial attack and plant defense. Proc. Natl. Acad. Sci. U.S.A. 106:10359-10364.

Pedley, K. F., and Martin, G. B. 2004. Identification of MAPKs and their possible MAPKK activators involved in the Pto-mediated defense response of tomato. J. Biol. Chem. 279:49229-49235.

Pedley, K. F., and Martin, G. B. 2005. Role of mitogen-activated protein kinases in plant immunity. Curr. Opin. Plant Biol. 8:541-547.

Pemberton, C. L., and Salmond, G. P. C. 2004. The Nep1-like proteins-A growing family of microbial elicitors of plant necrosis. Mol. Plant Pathol. 5:353-359.

Pemberton, C. L., Whitehead, N. A, Sebaihia, M., Bell, K. S., Hyman, L. J., Harris, S. J., Matlin, A. J. Robson, N. D., Birch, P. R. J., Carr, J. P., Toth, I. K., and Salmond, G. P. C. 2005. Novel quorum-sensing-controlled genes in Erwinia carotovora subsp. carotovora: Identification of fungal elicitor homologue in a soft-rotting bacterium. Mol. PlantMicrobe Interact. 18:343-353.

Pitzschke, A., and Hirt, H. 2006. Mitogen-activated protein kinases and reactive oxygen species signaling in plants. Plant Physiol. 141:351-356.

Popescu, S. C., Popescu, G. V., Bachan, S., Zhang, Z., Gerstein, M. Snyder, M., and Dinesh-Kumar, S. P. 2009. MAPK target networks in Arabidopsis thaliana revealed using functional protein microarrays. Genes Dev. 23:80-92.

Qutob, D., Kemmerling, B., Brunner, F., Küfner, I., Engelhardt, S., Gust, A. A., Luberacki, B., Seitz, H. U., Stahl, D., Rauhut, T., Glawischnig, E., Schween, G., Lacombe, B., Watanabe, N., Lam, E., Schlichting, R., Scheel, D., Nau, K., Dodt, G., Hubert, D., Gijzen, M., and Nürnberger, T. 2006. Phytotoxicity and innate immune responses induced by Nep1like proteins. Plant Cell 18:3721-3744.

Ren, D., Yang, H., and Zhang, S. 2002. Cell death mediated by MAPK is associated with hydrogen peroxide production in Arabidopsis. J. Biol. Chem. 277:559-565.

Ren, T., Zamboni, D. S., Roy, C. R., Dietrich, W. F., and Vance, R. E. 2006. Flagellin-deficient Legionella mutants evade caspase-1- and Naip5mediated macrophage immunity. PLoS Pathog. 2:e18. Published online.

Romeis, T., Piedras, P., Zhang, S. Q., Klessig, D. F., Hirt, H., and Jones, J. D. G. 1999. Rapid Avr9- and Cf-9-dependent activation of MAP kinases in tobacco cell cultures and leaves: Convergence of resistance gene, elicitor, wound, and salicylate responses. Plant Cell 11:273-287.

Rustérucci, C., Montillet, J. L., Agnel, J. P., Battesti, C., Alonso, B., Knoll, A., Bessoule, J. J., Etienne, P., Suty, L., Blein, J. P., and Triantaphylides, C. 1999. Involvement of lipoxygenase-dependent production of fatty acid hydroperoxides in the development of the hypersensitive cell death induced by cryptogein on tobacco leaves. J. Biol. Chem. 274:36446-36455.

Sagi, M., and Fluhr, R. 2001. Superoxide production by plant homologues of the gp91(phox) NADPH oxidase: Modulation of activity by calcium and by tobacco mosaic virus infection. Plant Physiol. 126:1281-1290.

Samuel, M. A., Hall, H., Krzymowska, M., Drzewiecha, K., Henning, J., and Ellis, B. E. 2005. SIPK signaling controls multiple components of harpin-induced cell death in tobacco. Plant J. 42:406-416.
Schouten, A., van Baarlen, P., and van Kann, J. A. 2008. Phytotoxic Nep1like proteins from the necrotrophic fungus Botrytis cinerea associate with membranes and the nucleus of plant cells. New Phytol. 177:493-505.

Schwessinger, B., and Zipfel, C. 2008. News from the frontline: Recent insights into PAMP-triggered immunity in plants. Curr. Opin. Plant Biol. 11:389-395.

Sharma, P. C., Ito, A., Shimizu, T., Terauchi, R., Kamoun, S., and Saitoh, H. 2003. Virus-induced silencing of WIPK and SIPK genes reduces resistance to a bacterial pathogen, but has no effect on the INF1-induced hypersensitive response (HR) in Nicotiana benthamiana. Mol. Genet. Genomics 269:583-591.

Staats, M., van Baarlen, P., Schouten, A., and van Kan, J. A. 2007. Func tional analysis of NLP genes from Botrytis elliptica. Mol. Plant Pathol. 8:209-214.

Takahashi, Y., Soyano, T., Sasabe, M., and Machida, Y. 2004. A MAP kinase cascade that controls plant cytokinesis. J. Biochem. 136:127-132.

Tavernier, E., Stallaert, V., Blein, J. P., and Pugin, A. 1995. Changes in lipid composition in tobacco cells treated with cryptogein, an elicitor from Phytophthora cryptogea. Plant Sci. 104:117-125.

Teige, M., Scheikl, E., Eulgem, T., Doczi, R., Ichimura, K., Shinozaki, K., Dangl, J.L., and Hirt, H. 2004. The MKK2 pathway mediates cold and salt stress signaling in Arabidopsis. Mol. Cell 15:141-152.

Tena, G., Asai, T., Chiu, W. L., and Sheen, J. 2001. Plant mitogen-activated protein kinase signaling cascades. Curr. Opin. Plant Biol. 4:392400.

Torres, M. A., Dangl, J. L., and Jones, J. D. G. 2002. Arabidopsis gp91 ${ }^{\text {phox }}$ homologues AtrbohD and AtrbohF are required for accumulation of reactive oxygen intermediates in plant defense response. Proc. Natl. Acad. Sci. U.S.A. 99:517-522.

Yamamoto, T. G., Chikashige, Y., Ozoe, F., Kawamukai, M., and Hiraoka, Y. 2004. Activation of the pheromone-responsive MAP kinase drives haploid cells to undergo ectopic meiosis with normal telomere clustering and sister chromatid segregation in fission yeast. J. Cell Sci. 117:3875-3886.

Yang, Y., Zhang, H., Li, G., Li, W., Wang, X., and Song, F. 2009. Ectopic expression of MgSM1, a Cerato-platanin family protein from Magnaporthe grisea, confers broad-spectrum disease resistance in Arabidopsis. Plant Biotechnol. J. 7:763-777.

Yoshioka, H., Numata, N., Nakajima, K., Katou, S., Kawakita, K., Rowland, O., Jones, J. D. G., and Doke, N. 2003. Nicotiana benthamiana gp $91^{\text {phox }}$ homologs $N$ brbohA and $N$ brbohB participate in $\mathrm{H}_{2} \mathrm{O}_{2}$ accumulation and resistance to Phytophthora infestans. Plant Cell 15:706-718.

Zhang, H. J., Fang, Q., Zhang, Z. G., Wang, Y. C., and Zheng, X. B. 2009 The role of respiratory burst oxidase homologs in elicitor-induced stomatal closure and hypersensitive response in Nicotiana benthamiana. J. Exp. Bot. 60:3109-3122.

Zhang, H. J., Dong, S. M., Wang, M. F., Wang, W., Song, W. W., Dou, X. Y., Zheng, X. B., and Zhang, Z. G. 2010. The role of vacuolar processing enzyme (VPE) from Nicotiana benthamiana in elicitor-triggered hypersensitive response and stomatal closure. J. Exp. Bot. 61:3799-3812.

Zhang, H. J., Wang, M. F., Wang, W., Li, D. Q., Huang, Q., Wang, Y. C., Zheng, X. B., and Zhang, Z. G. 2012. Silencing of G proteins uncovers diversified plant responses when challenged by three elicitors in Nicotiana benthamiana. Plant, Cell Environ. 35:72-85.

Zhang, S., and Klessig, D. F. 1998. The tobacco wounding-activated MAP kinase is encoded by SIPK. Proc. Natl. Acad. Sci. U.S.A. 95:74337438.

Zhang, S., and Klessig, D. F. 2001. MAPK cascades in plant defense signaling. Trends Plant Sci. 6:520-527.

Zhang, S., Du, H., and Klessig, D. F. 1998. Activation of the tobacco SIP kinase by both a cell wall-derived carbohydrate elicitor and purified proteinaceous elicitins from Phytophthora spp. Plant Cell 10:435-450.

Zhang, S., Liu, Y., and Klessig, D. F. 2000. Multiple levels of tobacco WIPK activation during the induction of cell death by fungal elicitins. Plant J. 23:339-347.

Zhou, B.-J., Jia, P.-S., Gao, F., and Guo, H.-S. 2012. Molecular characterization and functional analysis of a necrosis- and ethylene-inducing, protein-encoding gene family from Verticillium dahliae. Mol. PlantMicrobe Interact. 25:964-975. 